\title{
Tissue-specific exosome biomarkers for noninvasively monitoring immunologic rejection of transplanted tissue
}

\author{
Prashanth Vallabhajosyula, ${ }^{1}$ Laxminarayana Korutla, ${ }^{1}$ Andreas Habertheuer, ${ }^{1}$ Ming Yu, ${ }^{1}$ Susan Rostami, ${ }^{1}$ Chao-Xing Yuan, ${ }^{2}$ \\ Sanjana Reddy, ${ }^{1}$ Chengyang Liu, ${ }^{1}$ Varun Korutla, ${ }^{1}$ Brigitte Koeberlein, ${ }^{1}$ Jennifer Trofe-Clark, ${ }^{1}$ Michael R. Rickels,, ${ }^{3,4}$ and Ali Naji1,4 \\ Department of Surgery, ${ }^{1}$ Department of Pharmacology, Proteomics and Systems Biology Core, ${ }^{3}$ Department of Medicine, Division of Endocrinology, Diabetes and Metabolism, and ${ }^{4}$ Institute for Diabetes, \\ Obesity, and Metabolism, University of Pennsylvania Perelman School of Medicine, Philadelphia, Pennsylvania, USA.
}

\begin{abstract}
In transplantation, there is a critical need for noninvasive biomarker platforms for monitoring immunologic rejection. We hypothesized that transplanted tissues release donor-specific exosomes into recipient circulation and that the quantitation and profiling of donor intra-exosomal cargoes may constitute a biomarker platform for monitoring rejection. Here, we have tested this hypothesis in a human-into-mouse xenogeneic islet transplant model and validated the concept in clinical settings of islet and renal transplantation. In the xenogeneic model, we quantified islet transplant exosomes in recipient blood over long-term follow-up using anti-HLA antibody, which was detectable only in xenoislet recipients of human islets. Transplant islet exosomes were purified using anti-HLA antibody-conjugated beads, and their cargoes contained the islet endocrine hormone markers insulin, glucagon, and somatostatin. Rejection led to a marked decrease in transplant islet exosome signal along with distinct changes in exosomal microRNA and proteomic profiles prior to appearance of hyperglycemia. In the clinical settings of islet and renal transplantation, donor exosomes with respective tissue specificity for islet $\beta$ cells and renal epithelial cells were reliably characterized in recipient plasma over follow-up periods of up to 5 years. Collectively, these findings demonstrate the biomarker potential of transplant exosome characterization for providing a noninvasive window into the conditional state of transplant tissue.
\end{abstract}

\section{Introduction}

Immunologic rejection and immunosuppressive regimen-related complications remain the major causes of morbidity and mortality in transplant recipients. This is most evident in the fields of heart and lung transplantation, where the highest rates of immunologic rejection and patient mortality are seen despite routine surveillance biopsies to monitor organ status (1-4). In kidney transplantation, monitoring allograft rejection by rise in serum creatinine does not specifically portray immunologic rejection (5). In islet transplantation, where blood glucose monitoring remains the clinical standard, hyperglycemia typically heralds an advanced stage of rejection. Collectively, the current standards for monitoring transplant rejection reveal the critical need for more accurate, time-sensitive, and noninvasive biomarker platforms.

Several groups have reported on whole plasma/bodily fluid profiling of free nucleic acids and proteins as biomarker platforms for monitoring rejection, especially in the context of renal transplantation (6-17). But diagnostic accuracy remains a critical problem, as free nucleic acids and proteins are typically nonspecific and unstable in circulation, requiring a high steady state for reliable quantitation. Exosomes are extracellular vesicles released by many tissue types into bodily fluids, including blood, urine, and bronchoalveolar secretions. Exosomes represent stable and tissue-specific proteomic and

Conflict of interest: The authors have declared that no conflict of interest exists

Submitted: April 20, 2016; Accepted: January 24, 2017.

Reference information: J Clin Invest. 2017;127(4):1375-1391.

https://doi.org/10.1172/JCl87993.
RNA signature profiles that reflect the conditional state of their tissue of origin $(8-10,12,17)$. But similar to quantitative assays based on circulating free proteins and nucleic acids, whole plasma exosome analysis also introduces a high noise-to-signal ratio, as many tissue types contribute to the total plasma exosome pool. Therefore, quantitation and characterization of tissue-specific exosome profiles from bodily fluids would overcome this problem associated with whole plasma analysis, and would serve as a more accurate biomarker platform. In the context of transplantation, we hypothesized that transplanted tissue releases distinct, donor-specific exosomes into recipient plasma/bodily fluids, and its characterization would constitute a more accurate noninvasive biomarker platform for monitoring the conditional status of the transplanted organ. To quantify, purify, and characterize transplant exosomes and theirintraexosomal proteomic and RNA cargoes, we took advantage of 2 concepts: (a) exosomes express surface MHC antigens identical to their tissue counterparts, and (b) donor-recipient MHC mismatch introduced by transplantation enables characterization of transplant tissue-specific exosomes from recipient bodily fluids.

In this report, we detail our investigation of transplant tissuespecific exosome purification and characterization in an animal model of islet xenotransplantation (human into mouse), and validate the biomarker potential of this platform in the clinical settings of islet and renal transplantation.

\section{Results}

Transplanted human islets release donor $M H C$-specific exosomes into recipient plasma. First, we confirmed that exosomes released by 
A
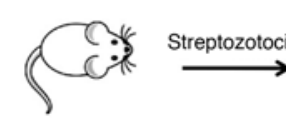

Athymic mouse

Diabetic nude mouse

Human islets

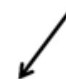

Xenoislet transplantation

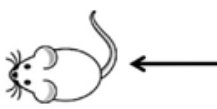

Normoglycemic nude mouse (follow-up 14 to 150 days)

C

Light scatter Fluorescence
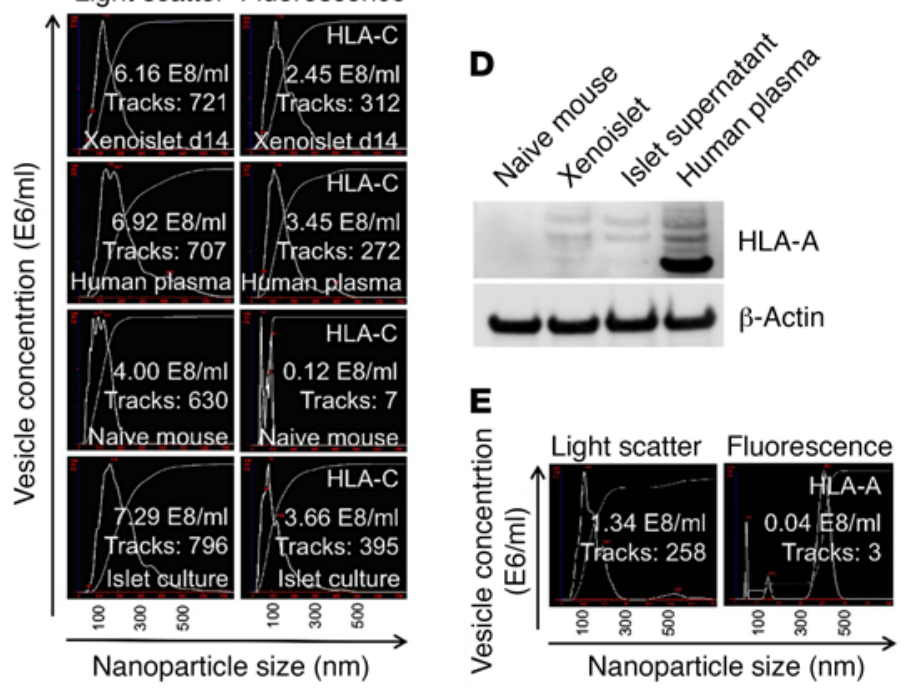

G

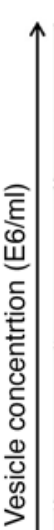

Light scatter Fluorescence

Tracks:
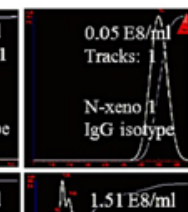

5,81 E8/nit

N-xeno 1

5:52 Es/ml

Tracks: 27

$\sqrt{1}$

1 N-xeno 2

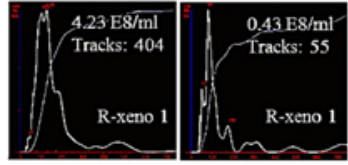

\& 8 \&
Light scatter Fluorescence
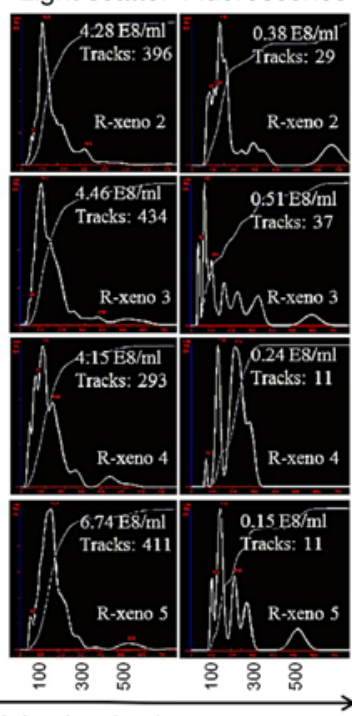

Nanoparticle size $(\mathrm{nm})$
B

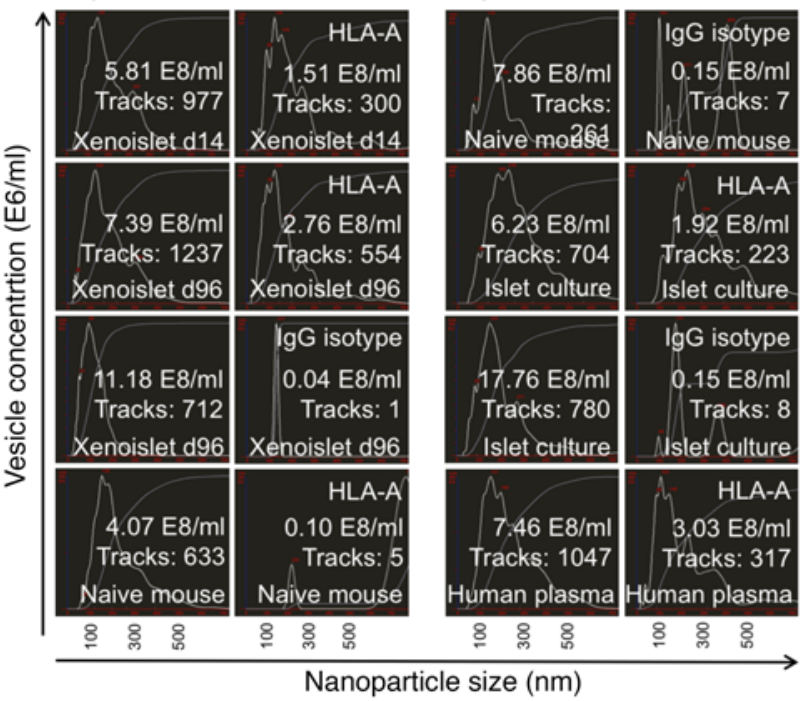

$\mathbf{F}$
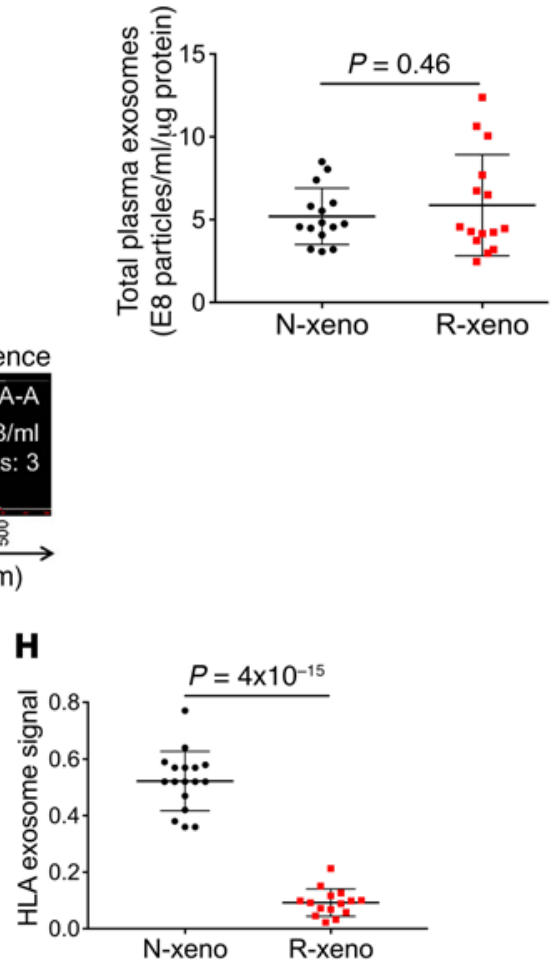
Figure 1. Transplanted human islets release donor-specific exosomes into recipient circulation. (A) Xenoislet transplantation model. Athymic mice were rendered diabetic and transplanted with human islets (2,000 islet equivalents) under the kidney capsule. Normoglycemia was monitored and recipients $(n=25)$ were sacrificed at various time points (days 14-150) for plasma exosome analysis. (B and C) Recipient plasma total exosome pool was analyzed on NanoSight nanoparticle detector on light scatter and fluorescence modes for donor islet-specific MHC signal using anti-HLA-A $(n=25)(B)$ and anti-HLA-C ( $n=10)($ C) quantum dot. All xenoislet samples $(n=25)$ showed donor HLA exosome signal compared with naive mouse $(n=25)$ and lgG isotype controls $\left(P=1.6 \times 10^{-14}\right)$. Representative samples from xenoislet post-transplant days 14 and 96 are shown. (D) Western blot analysis of total plasma exosomes showed HLA-A signal in xenoislet sample, but not naive mouse sample (1 of 4 shown). Positive controls included exosomes from human islet culture supernatant and human plasma. (E) NanoSight analysis of recipient plasma exosomes from day 14 after islet graftectomy for anti-HLA-A quantum dot was negative for TISEs $\left(P=1.4 \times 10^{-6}\right)$. Representative image from 1 of 6 animals is shown. (F) Total plasma exosome numbers were quantified on the NanoSight and expressed as number of nanoparticles per milliliter per microgram of exosome protein. Scatter plot with mean $\pm \mathrm{SD}$ for $\mathrm{N}$-xeno and R-xeno is shown ( $P=0.46, n=15$ in each study arm). (G) NanoSight analysis for HLA-A-positive exosomes showed signal reduction in all $\mathrm{R}$-xeno samples compared with $\mathrm{N}$-xeno animals receiving placebo (PBS) infusion. Representative results from $2 \mathrm{~N}$-xeno ( $\mathrm{N}$-xeno 1 and 2) and $5 \mathrm{R}$-xeno (R-xeno 1-5) animals and IgG isotype control from $\mathrm{N}$-xeno 1 are shown. (H) HLA exosome signal was significantly decreased in all the $\mathrm{R}$-xeno animals compared with $\mathrm{N}$-xeno $\left(P=4 \times 10^{-15}\right)$. Scatter plot with mean $\pm \mathrm{SD}$ is shown.

in vitro-cultured human islets into supernatant medium express human-specific MHC class I (HLA) antigens on their surface that are not detected on mouse plasma exosomes (Supplemental Figure 1; supplemental material available online with this article; https:// doi.org/10.1172/JCI87993DS1). We proposed that in the islet transplantation setting, islets would release HLA-specific exosomes into the recipient circulation compared with exosomes released by all other recipient tissues. To test our hypothesis, we used a xenogeneic islet transplantation model, where islets isolated from human pancreas are transplanted into athymic, diabetic nude mice (Figure 1A). Recipient animals were monitored for normoglycemia (Supplemental Figure 1), and their plasma exosomes were analyzed on the NanoSight nanoparticle detector fluorescence mode for HLA-positive exosomes (range 14-150 days; $n=25$ ). In all experiments, we confirmed that the majority of plasma extracellular microvesicles isolated using the methodology described were exosomes (Supplemental Figure 1 and ref. 18). At all tested time points, HLA-specific exosome signal (anti-HLA-A quantum dot) was detected in the xenoislet recipients, but this signal was undetectable in naive (nontransplanted) mouse plasma exosomes $\left(n=10,1.6 \times 10^{-14}\right)$ (Figure 1B and Supplemental Videos 1 and 2). HLA signal was also seen using antibody against another donor islet MHC marker, HLA-C (Figure 1C). Please refer to the Methods section for NanoSight exosome analysis shown for each panel. Further, Western blot analysis confirmed the NanoSight findings, as the xenoislet plasma exosomes showed the presence of HLA (Figure 1D). Next, we examined the specificity of the islet exosome HLA signal by performing transplant islet graftectomy $(n=6)$. This led to recurrence of severe hyperglycemia in the recipients, and on NanoSight fluorescence, there was complete loss of the donor HLA signal $\left(P=1.4 \times 10^{-6}\right.$; Figure 1E). Taken together, these data demonstrate that transplanted human islets release donor HLA-specific exosomes into recipient plasma that can be tracked over long-term follow-up, and this signal is specific to the transplanted human islet mass.

Given this, we then induced xenograft rejection in the recipients by inoculation with syngeneic leukocytes $(n=15$; Supplemental Figure 1). Recipients induced to reject the human islets are labeled as R-xeno in figures. Animals were sacrificed on the first day that the plasma glucose increased to more than $200 \mathrm{mg} / \mathrm{dl}$. Control recipients were injected with PBS and sacrificed 15 days later ( $n=15$; labeled as normoglycemic N-xeno in figures). On NanoSight, there were no quantitative differences in the total plasma exosome numbers between the 2 groups (Figure 1F), but the HLA exosome signal was markedly reduced in the R-xeno samples (Figure 1G). Quantitation of HLA signal demonstrated significant loss of plasma transplant islet exosomes $\left(P=4 \times 10^{-15}\right.$; Figure $1 \mathrm{H}$ ). Collectively, these data demonstrate that rejection leads to a quantifiable drop in transplant islet exosome signal that strongly correlated with the clinical picture of first-onset hyperglycemia and histologic evidence of islet rejection.

Plasma transplant islet exosome signal heralds acute rejection prior to detection of hyperglycemia. As the current standard for monitoring rejection in islet transplantation is based on glucose monitoring parameters, we further investigated the biomarker potential of the transplant exosome platform by performing a comparative analysis of the kinetics of the HLA exosome signal versus blood glucose monitoring during the evolution of rejection in the xenoislet model. In 2 independent experiments, donor sensitized leukocytes from syngeneic WT animals were infused into normoglycemic xenoislet recipients, and recipient plasma and islet graft were procured and analyzed at the following time points after leukocyte infusion ( $n=8$ animals per time point): days 0 (4 hours), 1, 2, 3, 5, and 7. Whereas the fasting blood glucose remained normal during the first 6 days after infusion of donor sensitized leukocytes, the HLA exosome signal significantly decreased by day 1 (Figure 2A; $\left.P=7.4 \times 10^{-7}\right)$, compared with the signal from day 0 , xenoislet, and placebo-infused time point control recipients. Further, over the next 5 days of the follow-up, the HLA exosome signal continued to decrease (Figure 2A and Supplemental Figure 1), whereas the total plasma exosome quantity was unchanged ( $P=0.62$ by 1 -way ANOVA; data not shown). The HLA exosome signal change was significantly different between the time points tested, and suggested a pattern of exponential decay in HLA exosome quantity (Figure 2A). In the xenoislet animals receiving placebo infusion, there was no difference in the HLA exosome signal between time points $(P=0.588$ by 1-way ANOVA). We also performed daily i.p. glucose tolerance tests on all animals and measured recipient plasma human-specific C-peptide levels for the time points tested to compare with the HLA exosome signal. Glucose tolerance tests remained normal through day 5 , and showed only mild impairment in glucose disposal on day 6 (Figure 2B), 1 day before the fasting blood glucose became elevated. Stimulated human C-peptide levels also remained within the normal range through day 5 (Supplemental Figure 1).

Given the significant drop in HLA exosome signal by the day 1 time point, we generated a receiver operating characteristic curve to understand its diagnostic potential to discriminate between 
A

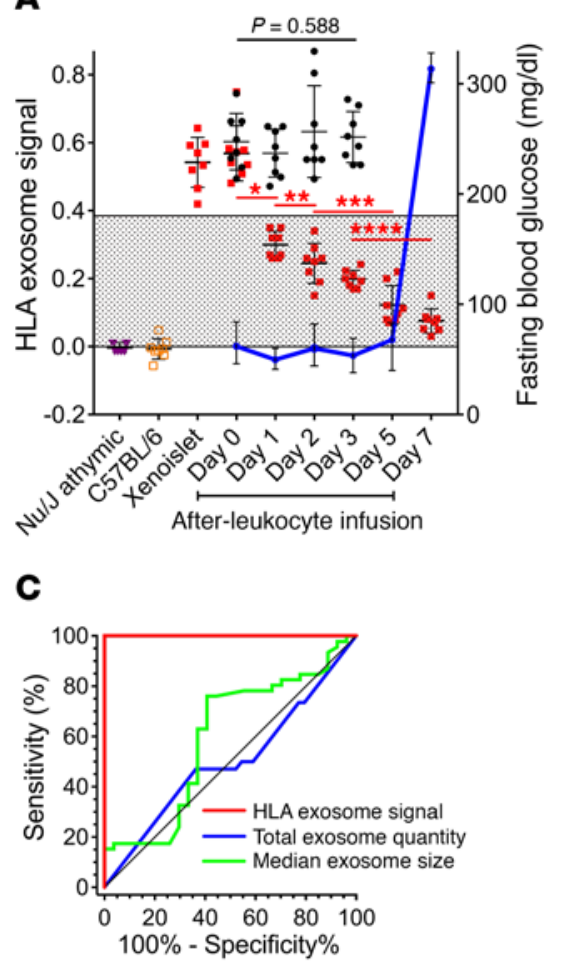

B
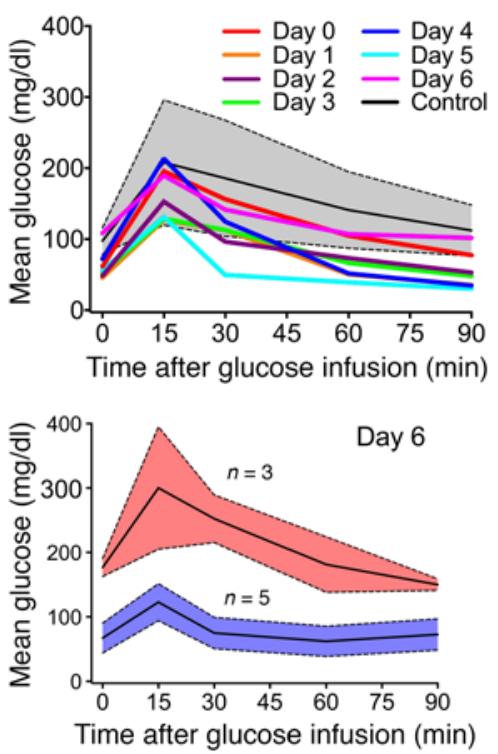

D
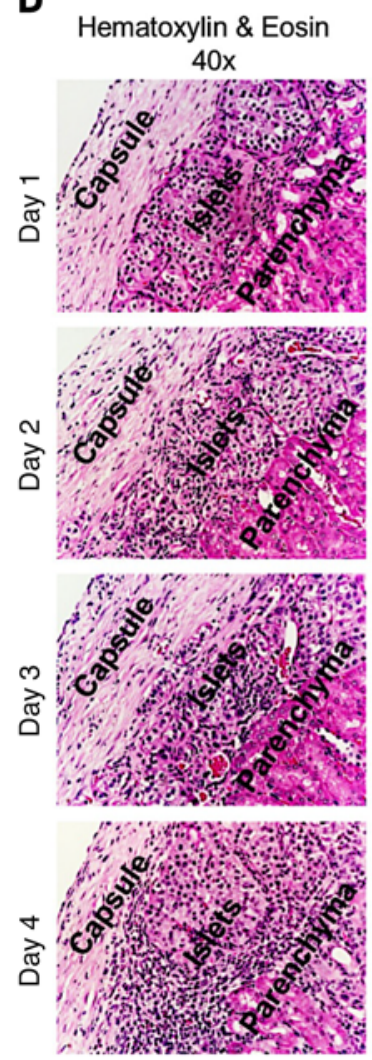

Immunohistochemistry
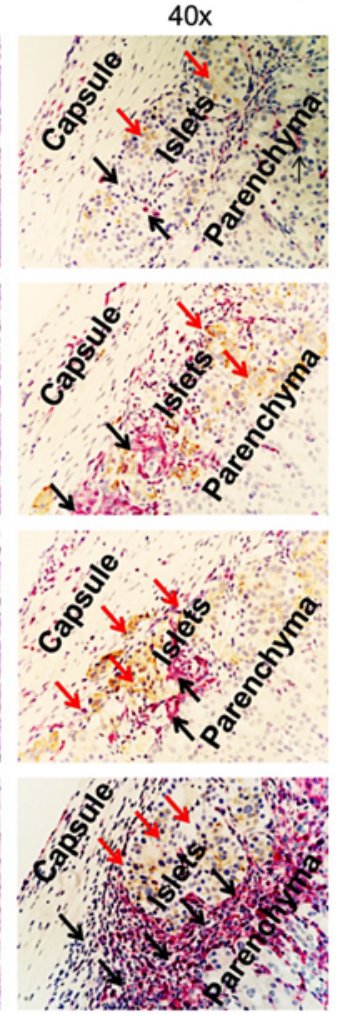

E

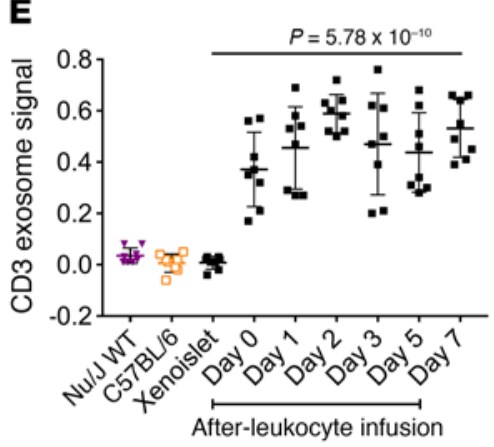

Figure 2. Transplant islet exosome profiles herald early acute rejection.

(A) Kinetics of HLA exosome signal in xenoislet recipients during evolution of acute rejection is shown in scatter plot. Donor-sensitized syngeneic leukocytes were infused to induce rejection, and HLA exosome signal (red, $y$ axis) was profiled at the following days: 0 (4 hours), 1, 2, 3, 5, and 7 ( $n=8$ animals per time point). The signal significantly decreased from day 0 to $1\left({ }^{*} P=7.4 \times 10^{-7}\right)$, day 1 to 2 (**P $=0.045)$, day 2 to 5 (***P=0.0008), and day 3 to $7\left({ }^{* * * *} P=1.7 \times 10^{-6}\right)$. Mean fasting blood glucose only increased on day 7 (blue line, secondary y axis). HLA exosome signal after placebo infusion (black; days 0-3) was similar between time points ( $n=8$ per time point, $P=0.588)$. Controls included athymic mouse, C57BL/6, and xenoislet ( $n=8$ for each). Summarized data (mean \pm SD) from 2 independent experiments are shown. (B) Daily i.p. glucose tolerance tests are shown (mean values, $n=8$ per time point). On day 6,3 of 8 animals showed impairment in glucose clearance (bottom). (c) Receiver operating characteristic curves for HLA exosome signal (red), total plasma exosome quantity (blue), and median exosome size (green) are shown. (D) Representative islet graft histology ( 1 of 4 ) is shown for days $1,2,3$, and 5 . H\&E and IHC for insulin (brown, red arrow) and T cells (CD3, pink, black arrow) are shown. On day 1 , viable islet clusters with very minimal $T$ cell infiltrate were seen. By day 5 , dense $T$ cell infiltration with islet destruction was seen, even though plasma glucose, glucose tolerance tests, and C-peptide levels were normal. (E) Plasma T cell exosome signal (CD3 signal) is shown (mean $\pm \mathrm{SD}$ ). Compared with xenoislet, Nu/J WT, and third-party C57BL/ 6 controls, samples from day 0 to 7 showed persistently elevated CD3 exosome signal ( $n=8$ per time point, $\left.P=5.78 \times 10^{-10}\right)$. no rejection (xenoislet, $\mathrm{N}$-xeno, placebo infusion, and day 0 time points, $n=80$ ) and rejection (day 1 or greater time points, $n=40$ ). This showed an AUC of $1.0 \pm 0.0$, with sensitivity and specificity of $100 \%$ for signal threshold cutoff of 0.38 (Figure $2 \mathrm{C}$ ). AUC values for total exosome quantity (AUC $0.50 \pm 0.07$ ) and median exo- some size (AUC $0.56 \pm 0.07$ ) showed poor ability to discriminate for rejection using these parameters. To further correlate these findings with the acute rejection process at the tissue level, we performed islet graft histology (Figure 2D). Day 1 histology showed few infiltrating CD3-positive $\mathrm{T}$ cells around viable islet clusters, 
although the HLA exosome signal was already significantly lower by this time point. By day 5, progressive $\mathrm{T}$ cell infiltration with islet destruction was evident on histology, when fasting glucose, glucose tolerance test, and human C-peptide were still within the normal range. These findings support that, unlike glucose monitoring, the decay in plasma HLA exosome signal correlates with early changes in the acute rejection process, when there is minimal $\mathrm{T}$ cell infiltrate without islet destruction. Collectively, these data demonstrate that in the xenoislet model, transplant islet exosome profiling is a more accurate and time-sensitive noninvasive biomarker of acute rejection than current standards such as fasting glucose, glucose tolerance test, and human C-peptide analysis.

Next, we hypothesized that acute rejection of islets would also lead to sustained elevation in recipient plasma $\mathrm{T}$ cell exosome signal, as stimulated xenoreactive T cells would release a steady pool of exosomes into the plasma. A combined analysis of donor MHC-specific exosome signal and recipient T cell exosome characterization may further increase the accuracy of the proposed biomarker platform. T cell exosome signal was quantified using anti-CD3 antibody. Compared with naive WT strain-matched $(\mathrm{Nu} / \mathrm{J})$, third-party $\mathrm{C} 57 \mathrm{BL} / 6$, and xenoislet controls, acute rejection animals from day 0 showed a quantifiable CD3 exosome signal in the recipient plasma, which remained elevated during the entire rejection process (Figure $2 \mathrm{E}$ ). Taken together, this suggests that a combined plasma tissue-specific exosome profiling (donor HLA, recipient $\mathrm{T}$ cell and B cell) may provide a time-sensitive noninvasive window into the rejection-specific interactions between the transplant tissue and the recipient immune system.

Donor-recipient MHC mismatch enables purification of human islet cell-specific exosomes from recipient plasma. Next we sought to determine whether rejection would cause distinct changes in the intra-exosomal proteomic and RNA cargoes of transplant islet exosomes and, if so, whether its characterization would further improve the diagnostic accuracy of the platform. To purify transplant islet-specific exosomes (TISEs), we used anti-HLA-A-specific affinity antibody beads to obtain an HLA-A-bound fraction (representing TISEs) and an HLA-A-unbound fraction (representing recipient mouse tissue exosomes) (Supplemental Figure 2). First, to validate that the antibody beads were binding intact TISEs and not freely circulating plasma protein aggregates, we performed transmission electron microscopy of eluted HLA-A-bound exosomes. Distinct, intact exosomes (40-to-100-nm range) were noted on electron microscopy (Figure 3A). Next, to validate optimal capture of TISEs, we confirmed that the HLA-A-unbound fraction in the xenoislet sample was negative for HLA-A surface expression on NanoSight fluorescence and on Western blot (Supplemental Figure 2). We also confirmed that there was not a major contribution from donor passenger leukocytes into the HLA-A-bound exosome fraction (Supplemental Figure 2). Taken together, this suggested that the majority of donor exosomes were human islet cell derived.

Given this, we assessed whether HLA-A-bound exosomes coexpress surface markers specific for islet endocrine cellular constituents. This would also validate that the antibody beads were binding intact exosomes. The transmembrane protein ion channel regulator FXYD2 isoforms $\gamma \mathrm{a}$ and $\gamma \mathrm{b}$ is reported to be an islet $\beta$ cell-specific surface marker compared with exocrine pancreas (19-22). Therefore, we assessed for FXYD2 surface coexpression in the HLA-A-bound exosomes as an indicator that we were purifying human islet $\beta$ cell-specific exosomes. On NanoSight fluorescence, HLA-A-enriched xenoislet and human islet culture supernatant exosome samples were highly positive for coexpression of FXYD2, but not human plasma and naive mouse plasma samples (Figure 3B and Supplemental Videos 3 and 4). Naive human plasma HLA-A-bound exosomes were analyzed to confirm that FXYD2 coexpression was specific to the human islet exosomes, not all HLA-A-expressing human-derived exosomes.

Next, given the mounting evidence for functional roles of exosomes, we tested whether TISEs carried islet endocrine hormones as part of their cargo. Xenoislet recipient and human islet culture supernatant samples showed expression of insulin, glucagon, and somatostatin on Western blot (Figure 3C). Western blot also showed expression of FXYD2 only in the xenoislet and islet supernatant fractions (Figure 3C), validating the NanoSight findings (Figure 3B). Further, reverse transcriptase PCR (RT-PCR) showed islet endocrine hormone mRNAs and FXYD2 mRNA in the xenoislet and human islet culture supernatant samples, but not in naive mouse plasma sample (Figure 3D). To confirm that these findings were not falsely positive due to HLA-A antibody beads nonspecifically binding plasma free forms of islet endocrine hormones, we tested the plasma of normal human subjects $(n=5)$ and noted absence of islet endocrine hormones on Western blot and RT-PCR analyses (Figure $3, \mathrm{C}$ and $\mathrm{D} ; P=0.007)$. Collectively, these data demonstrated that we were enriching bona fide transplant islet exosomes that express islet-specific surface markers, and carry islet endocrine hormones as part of their intra-exosomal cargo. Transplant tissue-specific exosomes can be purified from recipient plasma.

Immune rejection leads to a decrease in endocrine hormone signatures in islet exosomes. Having demonstrated quantitative changes in TISEs with islet graftectomy and immune rejection, we studied whether these conditions accordingly lead to changes in their endocrine hormone expression. On NanoSight fluorescence, HLA-A-bound fraction from islet graftectomy plasma samples did not show FXYD2 coexpression (Figure 3E); and on Western blot FXYD2 and insulin were undetectable (Figure 3F). Therefore, the expression of islet endocrine hormones was transplant tissue specific. Given this, we assessed for TISE expression of islet endocrine hormones under conditions of immune rejection (R-xeno). On NanoSight fluorescence, R-xeno samples showed decreased coexpression of FXYD2 (Figure 3G). On Western blot and RT-PCR, a very faint signal for insulin and FXYD2 was detected in comparison with the N-xeno sample (Figure 3, H and I). Collectively, these data indicate that immune rejection leads to decreased detection of islet endocrine cell markers in the HLA-A-bound exosome fraction.

Islet exosomes express distinct protein and RNA signatures compared with the transplanted islets. We assessed an in vivo readout of TISE cargo as compared with its transplanted human islet tissue counterpart. Transplant islet exosomes from 2 independent experiments were analyzed on mass spectrometry for proteomic profiling (Figure 4A). A majority of proteins were commonly expressed in both samples (Figure 4A). Detailed results of mass spectrometry from 1 experiment are shown in Supplemental Table 1. Next, long and small RNA microarray profiling of islet exosomes and islet graft tissue was performed. TISEs were rich in small RNAs $(<30$ nucleotides), with minimal ribosomal RNA, unlike the transplanted 
A
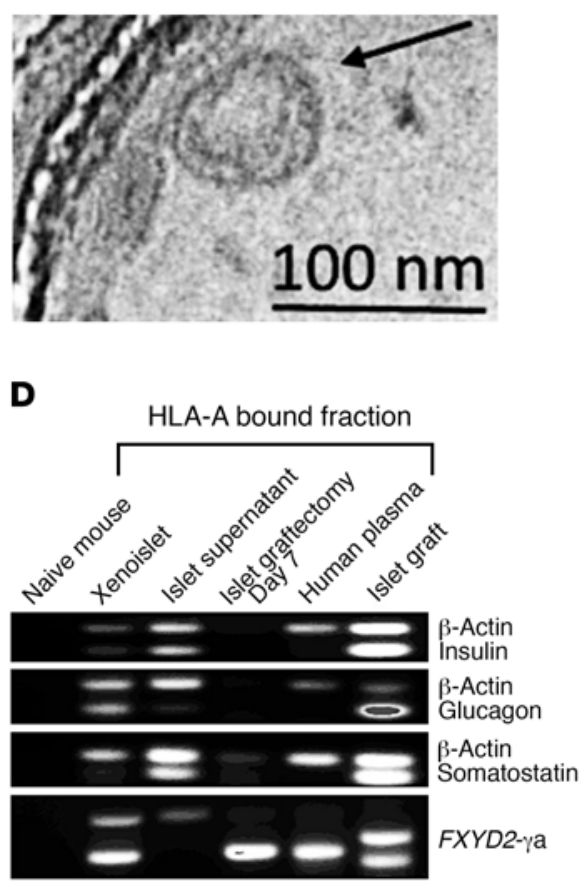

E

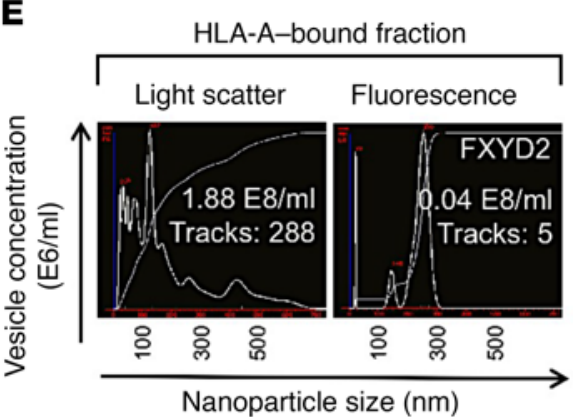

B

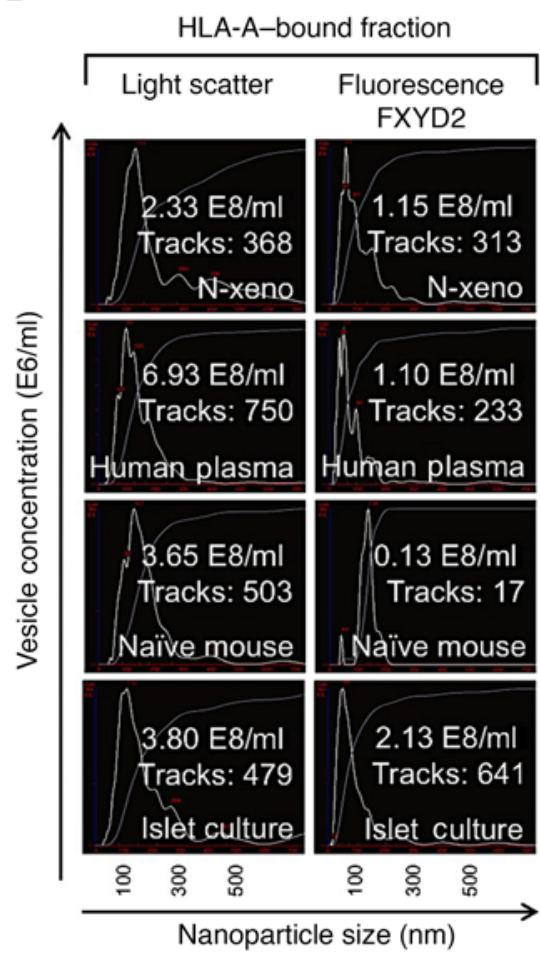

$\mathbf{F}$

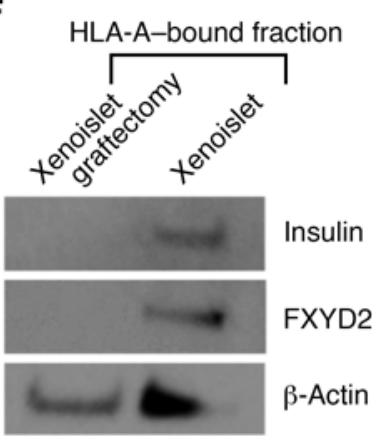

C

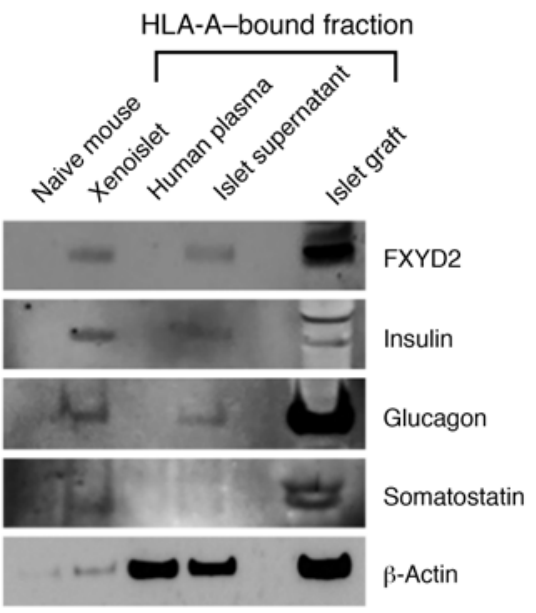

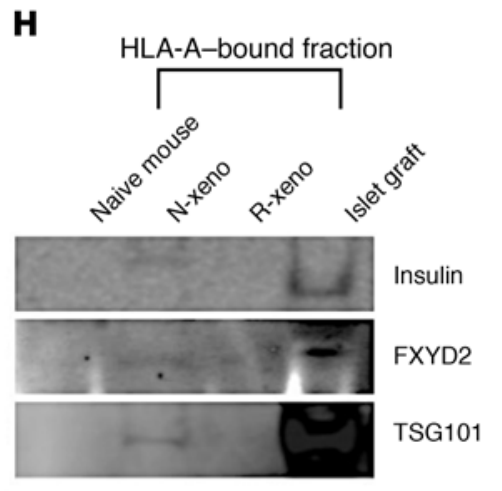

I

HLA-A-bound fraction
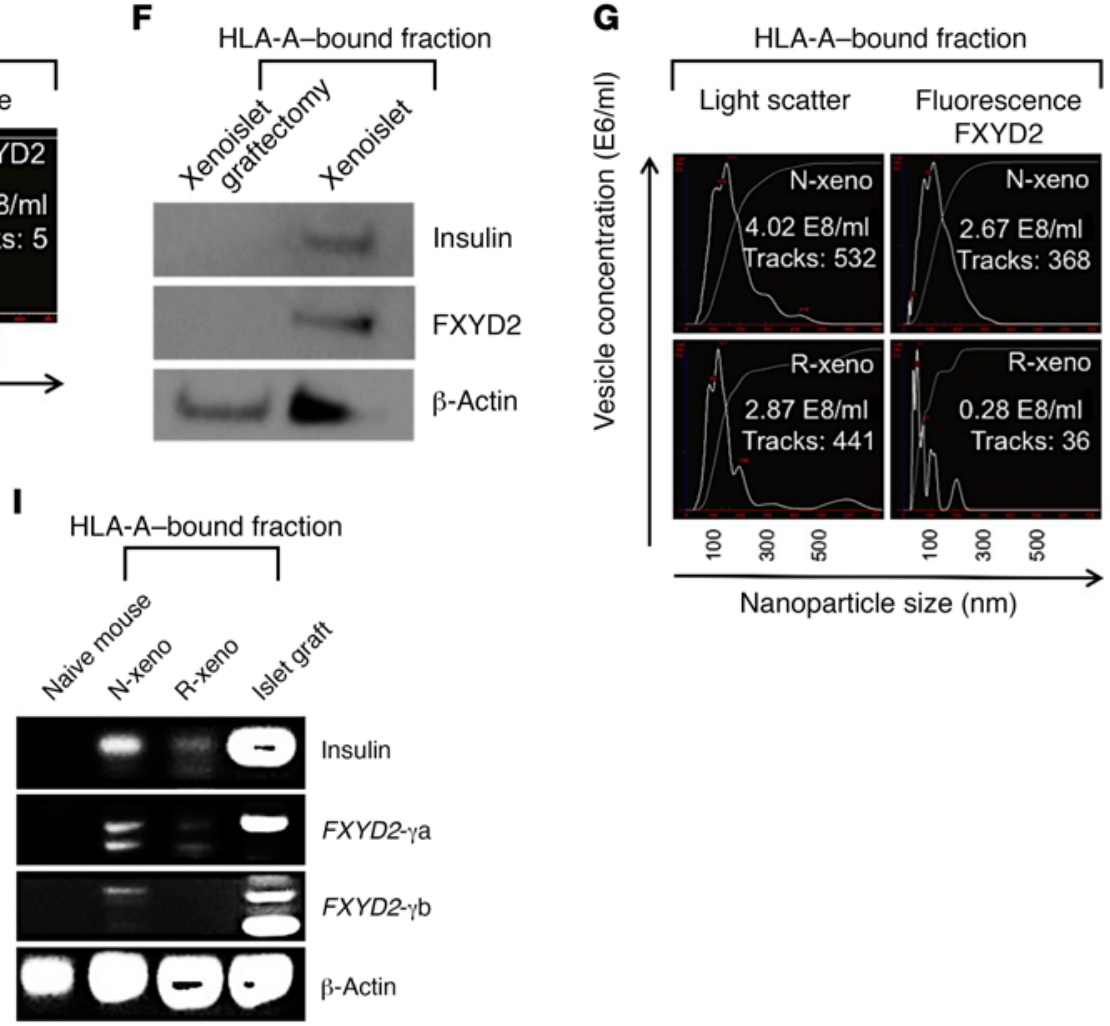
Figure 3. Transplant islet exosome purification and characterization of its intra-exosomal cargo. (A) Transmission electron microscopy of HLA-A antibody-bound content from xenoislet plasma exosomes showed that intact exosomes (range 40-100 nm; arrow) were being enriched. (B) NanoSight analysis of HLA-A-bound exosome fraction for coexpression of the $\beta$ cell marker FXYD2 showed high expression only in N-xeno and human islet culture samples, but not in human plasma or naive mouse plasma HLA-A-bound fractions ( $P=0.008 ; 1$ of 5 experiments shown). (C and $\mathbf{D})$ Western blot (C) and RT-PCR (D) analysis of HLA-A-bound exosomes showed presence of FXYD2 and islet endocrine hormones from xenoislet plasma and human islet culture supernatant samples, but not human plasma and naive mouse plasma (negative controls) samples $(P=0.008 ; 1$ of 5 experiments shown). Islet graft served as positive control. (E and F) Plasma HLA-A-bound exosomes 14 days after islet graftectomy $(n=6)$ showed low surface coexpression of FXYD2 on NanoSight fluorescence (E). Western blot analysis showed absence of insulin and FXYD2 proteins in this fraction (F). One of six experiments is shown $(P=0.002)$. (G-I) HLA-A-bound exosomes from R-xeno plasma showed decreased coexpression of FXYD2 on NanoSight fluorescence (G). Western blot (H) and RT-PCR (I) analysis showed decreased levels of FXYD2 and insulin proteins and mRNAs compared with $\mathrm{N}$-xeno sample $(P=0.002)$. Controls include naive mouse HLA-A-bound content and xenoislet graft tissue. One of six experiments is shown.

islet tissue (Figure 4B). Microarray for long RNA further validated mRNA expression of insulin, glucagon, somatostatin, and FXYD2 (Figure 4C). Although the highest-expressing long RNAs between the 2 samples were similar, small RNA profiles showed distinct enrichment of microRNAs in TISEs. The 25 highest-expressing microRNAs in TISEs and their relative levels in islet graft and vice versa are shown in Table 1. Only 4 microRNAs were commonly expressed at the highest level in both groups: miR-191-5p, miR-23a3p, miR-16-5p, and miR-24-3p. Also, the 25 highest upregulated microRNAs in TISEs compared with islet graft (highest enrichment) and vice versa are shown in Table 2. In this comparison, miR-375, a highly expressed $\beta$ cell-specific microRNA $(23,24)$, was 3,285 fold enriched in islet graft. miR-122-5p, reported to be liver specific
(25-30), was 1,060-fold enriched in TISEs. Taken together, these data demonstrate that anti-donor HLA-specific exosome purification from recipient plasma enables detailed characterization of the proteomic and RNA signatures of transplant islet exosomes.

Rejection leads to distinct changes in TISE cargo. Having demonstrated a significant drop in TISE numbers with rejection, we assessed whether this leads to changes in TISE proteomic and RNA profiles (N-xeno versus $\mathrm{R}$-xeno). Proteomic profiles from 3 independent $\mathrm{R}$-xeno experiments were compared with the $2 \mathrm{~N}$-xeno samples to look for consistent differences between rejection and normal conditions. We selected proteins that were either absent or expressed at very low levels in the $2 \mathrm{~N}$-xeno samples compared with the $3 \mathrm{R}$-xeno samples, and vice versa. We confirmed that the

Table 1. Top 25 highest-expressing microRNAs in TISE and islet graft tissue from a normoglycemic xenoislet animal

Islet graft

TISE

\section{MicroRNA}

hsa-miR-8075

hsa-miR-3613-3p

hsa-miR-4668-5p

hsa-miR-5787

hsa-miR-4508

hsa-miR-6732-5p

hsa-miR-486-5p

hsa-miR-3613-5p

hsa-miR-1281

hsa-miR-7704

hsa-miR-1469

hsa-miR-4787-5p

hsa-miR-638

hsa-miR-6803-5p

hsa-miR-455-3p

hsa-miR-4466

hsa-miR-93-5p

hsa-miR-1825

hsa-miR-3201

hsa-miR-1237-5p

hsa-miR-3656

hsa-miR-4529-3p

hsa-miR-17-5p

hsa-miR-8069
hsa-miR-762
Expression

\begin{tabular}{cc}
5783.0 & 114.8 \\
5674.6 & 57.8 \\
5452.2 & 66.3 \\
5220.3 & 8.7 \\
5204.4 & 9.3 \\
4776.7 & 70.0 \\
4093.8 & 13.9 \\
3950.7 & $1,666.8$ \\
3901.0 & 85.5 \\
3898.0 & 6.3 \\
3836.1 & 5.5 \\
\hline 3717.5 & 4.8 \\
3547.1 & 6.3 \\
\hline 3520.9 & 4.5 \\
\hline 3403.3 & 35.3 \\
3296.9 & 4.3 \\
3242.1 & 3.1 \\
\hline 3181.1 & 164.2 \\
\hline 3137.5 & 604.0 \\
\hline 3053.4 & 7.2 \\
\hline 3049.0 & 4.3 \\
\hline 3031.3 & $1,073.2$ \\
\hline 2977.1 & 9.8 \\
\hline 2948.6 & 3.4 \\
\hline 2724.9 & 4.6 \\
\hline
\end{tabular}

MicroRNA

hsa-let-7c-5

hsa-let-7b-5p

hsa-miR-26a-5p

hsa-let-7d-5p

hsa-let-7a-5p

hsa-miR-16-5p

hsa-miR-23b-3p

hsa-miR-24-3p

hsa-miR-125b-5p

hsa-miR-22-3p

hsa-miR-23a-3p

hsa-miR-103a-3p

hsa-miR-194-5p

hsa-miR-107

hsa-miR-206

hsa-miR-191-5p

hsa-miR-30c-5p

hsa-let-7i-5p

hsa-miR-126-3p

hsa-miR-378a-3p

hsa-miR-145-5p

hsa-miR-375

hsa-miR-192-5p

hsa-miR-29a-3p

hsa-let-7e-5p

\begin{tabular}{cc} 
Expression & $\begin{array}{c}\text { Islet graft expression/ } \\
\text { TISE expression }\end{array}$ \\
\hline 8985.5 & 2.9 \\
7073.9 & 2.3 \\
6210.9 & 13.8 \\
\hline 4707.9 & 10.5 \\
\hline 4483.4 & 263.0 \\
\hline 4315.4 & 1.2 \\
\hline 4258.1 & 5.0 \\
\hline 4011.4 & 1.2 \\
\hline 3644.2 & 213.8 \\
\hline 3545.8 & 38.7 \\
\hline 3542.8 & 0.9 \\
\hline 3485.6 & 6.7 \\
\hline 3482.2 & $3,914.1$ \\
\hline 3078.4 & 5.4 \\
\hline 2775.6 & $2,607.2$ \\
\hline 2714.9 & 0.6 \\
\hline 2680.9 & $1,635.9$ \\
\hline 2490.9 & 27.2 \\
\hline 2466.2 & $2,772.1$ \\
\hline 2464.5 & 561.0 \\
\hline 2406.3 & 13.7 \\
\hline 2354.5 & $3,284.9$ \\
\hline 2304.1 & 268.8 \\
\hline 2207.8 & 5.6 \\
\hline 2102.9 & $2,752.0$ \\
\hline $115 E$ & \\
\hline
\end{tabular}

Expression value for microRNA was normalized to the median value for that microarray. The relative expression in TISE compared with islet graft (TISE/ islet graft) and vice versa is also shown. 
Table 2. Specific microRNAs are upregulated in TISE compared with the transplant islet graft

\begin{tabular}{|c|c|c|c|}
\hline MicroRNA & $\begin{array}{l}\text { Fold upregulation in TISE } \\
\text { (TISE/islet graft) }\end{array}$ & MicroRNA & $\begin{array}{l}\text { Fold downregulation in TISE } \\
\text { (- Islet graft/TISE) }\end{array}$ \\
\hline hsa-miR-3613-5p & $1,666.8$ & hsa-miR-194-5p & $-3,914$ \\
\hline hsa-miR-4529-3p & $1,073.2$ & hsa-miR-375 & $-3,285$ \\
\hline hsa-miR-122-5p & $1,059.7$ & hsa-miR-126-3p & $-2,772$ \\
\hline hsa-miR-3201 & 604.0 & hsa-let-7e-5p & $-2,752$ \\
\hline hsa-miR-4487 & 552.4 & hsa-miR-206 & $-2,607$ \\
\hline hsa-miR-3128 & 463.8 & hsa-miR-30a-5p & $-2,004$ \\
\hline hsa-miR-3157-3p & 270.4 & hsa-miR-199a-3p & $-1,693$ \\
\hline hsa-miR-4423-3p & 265.8 & hsa-miR-199b-3p & $-1,693$ \\
\hline hsa-miR-6797-3p & 252.9 & hsa-miR-30c-5p & $-1,636$ \\
\hline hsa-miR-3921 & 248.2 & hsa-miR-99a-5p & $-1,584$ \\
\hline hsa-miR-4750-3p & 239.7 & hsa-miR-127-3p & $-1,526$ \\
\hline hsa-miR-6777-5p & 202.4 & hsa-miR-200a-3p & $-1,313$ \\
\hline hsa-miR-7108-3p & 192.4 & hsa-miR-151a-5p & $-1,274$ \\
\hline hsa-miR-8084 & 188.4 & hsa-let-7f-5p & $-1,231$ \\
\hline hsa-mir-6776 & 179.0 & hsa-miR-200c-3p & $-1,213$ \\
\hline hsa-miR-4433-5p & 168.3 & hsa-let-7g-5p & $-1,187$ \\
\hline hsa-miR-1825 & 164.2 & hsa-miR-195-5p & $-1,164$ \\
\hline hsa-miR-520g & 156.7 & hsa-miR-214-3p & $-1,058$ \\
\hline hsa-miR-520h & 156.7 & hsa-miR-181a-5p & $-1,055$ \\
\hline hsa-miR-940 & 156.1 & hsa-miR-199a-5p & $-1,041$ \\
\hline hsa-miR-191-3p & 155.9 & hsa-miR-652-3p & $-1,002$ \\
\hline hsa-miR-5571-5p & 147.0 & hsa-miR-200b-3p & -949 \\
\hline hsa-miR-4310 & 136.6 & hsa-miR-27b-3p & -943 \\
\hline hsa-miR-1228-3p & 133.2 & hsa-miR-152-3p & -847 \\
\hline hsa-miR-4655-5p & 118.4 & hsa-miR-10a-5p & -832 \\
\hline
\end{tabular}

identified proteins were human derived, not mouse derived, by comparing all peptide matches for a given protein on NCBI Protein BLAST against human and mouse forms of the protein of interest. We also searched ExoCarta (www.exocarta.org) and confirmed that the proteins of interest have been reported by other groups to be expressed in exosomes. Subsequently, 4 human proteins showed consistent expression differences between normal and rejection conditions: heat shock cognate protein 70 (HSC70), angiopoietin-1, hemopexin, and complement C3 (Figure 5, A-D).

Next, we attempted small RNA profiling of R-xeno samples, but because of the low TISE levels from a single sample we had to pool transplant islet exosomes from 5 animals for microarray analysis. We compared microRNAs from R-xeno sample that showed at least 2-fold or greater expression over the median value. The list of microRNAs differentially upregulated with immune rejection is shown in Table 3. Collectively, these data demonstrate that immune rejection alters the proteomic and RNA cargoes of TISEs, and these immune rejection-specific changes attest to the biomarker potential of this noninvasive platform.

Clinical islet allotransplantation. Given validation of the concept in an animal model, we sought to determine whether transplant islet exosomes could be detected in the clinical setting. We obtained plasma samples from 5 type 1 diabetic recipients enrolled in the NIH Clinical Islet Transplantation Consortium trial. Donor islet exosomes were characterized from recipient plasma samples during pretransplant, peritransplant, and long-term post-transplant follow-up (up to 5 years) using anti-donor HLA class I-specific alloantibodies. Donor-recipient HLA profiles and clinical data (fasting serum glucose, C-peptide, type 1 diabetes autoantibody levels) for patients $\mathrm{A}$ to D are shown in Supplemental Tables 2-5. In all recipients, donor islet exosomes were undetectable before transplant, but quantifiable at every tested post-transplant time point $(P=0.0001)$. NanoSight panels for the donor islet exosome signals for patients A to D are shown in Supplemental Figure 3. Graphical presentation of donor HLA class I-specific exosome signal and recipient plasma C-peptide-to-glucose ratio over long-term follow-up is shown in Figure 6, A-D. In 3 patients (B-D), the donor HLA exosome signal remained stable over follow-up, but in patient A, a drop in the signal to less than 0.35 was noted in the sample available from the day 1,001 time point when the C-peptide-to-glucose ratio was still normal. At the same time this patient demonstrated a significant rise of de novo autoantibody against the $\beta$ cell-specific antigen glutamic acid decarboxylase 65 (GAD65) (Figure 6E); and by day 1,197 the recipient had onset of hyperglycemia without evidence of alloantibody formation (31), supporting recurrence of autoimmune diabetes in this subject (Supplemental Table 2). Thus, in patient A the decrease in transplant islet exosome signal temporally correlated with the recurrence of islet autoimmunity, which preceded the clinical onset of hyperglycemia. Taken together, these findings demonstrate that transplant islet exosomes can be reliably quantified over long-term follow-up in patients undergoing islet allotransplantation, and donor HLA exosome signal changes may reflect early injury/loss of islet mass.

Next we tested whether TISE cargoes can be purified and characterized in the clinical setting. Representative results from patient $\mathrm{E}$ are shown ( 1 of 5) (Figure 7). This patient experienced loss of $\beta$ cell function due to accelerated acute rejection in the peritransplant period (Figure 7A). First, we confirmed that all tested plasma samples were enriched in exosomes (Figure 7B). Next, using donorspecific HLA-A2 antibody we confirmed that transplant islet exosomes were detectable after transplant, but not before transplant (Figure 7C). Transplant islet exosomes were highly positive for coexpression of FXYD2 (Figure 7D) and showed expression of insulin, glucagon, somatostatin, and FXYD2 proteins in posttransplant day 2 plasma sample (Figure 7E), correlating with correction of hyperglycemia in the patient (Figure 7A). RNA cargo analysis showed expression of insulin, glucagon, somatostatin, and FXYD2, which was undetectable in the pretransplant sample (Figure $7 \mathrm{~F}$ ). Plasma analysis for donor exosomes was negative for HLA-A2 sig- 


\section{Table 3. Immune rejection of transplanted islets changes the microRNA profiles in TISE}

\begin{tabular}{|c|c|c|c|}
\hline MicroRNA & Expression R-Xeno & Expression $\mathrm{N}$-Xeno & Fold enrichment \\
\hline hsa-miR-4722 & 7.5 & 1.1 & 6.7 \\
\hline hsa-miR-4722-3p & 9.5 & 1.8 & 5.3 \\
\hline hsa-miR-6764-5p & 4.1 & 0.9 & 4.6 \\
\hline hsa-miR-516a-1 & 1.8 & 0.6 & 3.1 \\
\hline hsa-miR-516a-2 & 1.8 & 0.6 & 3.1 \\
\hline hsa-miR-5093 & 2.1 & 0.7 & 3.0 \\
\hline hsa-miR-4786-3p & 3.2 & 1.1 & 2.9 \\
\hline hsa-miR-4281 & 7.5 & 2.6 & 2.8 \\
\hline hsa-miR-8087 & 1.4 & 0.5 & 2.6 \\
\hline hsa-miR-4656 & 2.1 & 0.8 & 2.6 \\
\hline hsa-miR-1225 & 1.6 & 0.7 & 2.5 \\
\hline hsa-miR-5681b & 1.9 & 0.8 & 2.4 \\
\hline hsa-miR-135b & 1.5 & 0.6 & 2.3 \\
\hline hsa-miR-5683 & 1.6 & 0.7 & 2.3 \\
\hline hsa-miR-4755 & 1.5 & 0.6 & 2.3 \\
\hline hsa-miR-4729 & 1.5 & 0.6 & 2.3 \\
\hline hsa-miR-4481 & 1.5 & 0.6 & 2.2 \\
\hline hsa-miR-1229-5p & 1.6 & 0.7 & 2.2 \\
\hline hsa-miR-3184-3p & 2.1 & 1.0 & 2.2 \\
\hline hsa-miR-1307-3p & 1.6 & 0.8 & 2.2 \\
\hline hsa-miR-573 & 1.6 & 0.8 & 2.2 \\
\hline hsa-miR-570 & 1.6 & 0.8 & 2.1 \\
\hline hsa-miR-4720-3p & 1.6 & 0.8 & 2.1 \\
\hline hsa-miR-4642 & 1.5 & 0.7 & 2.1 \\
\hline hsa-miR-3115 & 1.5 & 0.7 & 2.1 \\
\hline hsa-miR-6759 & 1.6 & 0.8 & 2.1 \\
\hline hsa-miR-5001-5p & 1.6 & 0.8 & 2.1 \\
\hline hsa-miR-4665 & 1.6 & 0.8 & 2.0 \\
\hline hsa-miR-3187-5p & 1.9 & 0.9 & 2.0 \\
\hline hsa-miR-3137 & 1.6 & 0.8 & 2.0 \\
\hline hsa-miR-4776-3p & 1.6 & 0.8 & 2.0 \\
\hline hsa-miR-6768-5p & 1.5 & 0.7 & 2.0 \\
\hline hsa-miR-4739 & 1.5 & 0.7 & 2.0 \\
\hline hsa-miR-513c-3p & 1.5 & 0.7 & 2.0 \\
\hline hsa-miR-7855-5p & 1.9 & 0.9 & 2.0 \\
\hline hsa-miR-6835 & 1.6 & 0.8 & 2.0 \\
\hline hsa-miR-664a-3p & 1.5 & 0.7 & 2.0 \\
\hline hsa-miR-4771 & 1.5 & 0.7 & 2.0 \\
\hline hsa-miR-3689a & 1.6 & 0.8 & 2.0 \\
\hline
\end{tabular}

Small RNA profiling data from TISEs enriched from R-xeno sample versus $\mathrm{N}$-xeno sample were compared. MicroRNAs with more than 2 -fold expression over median value from R-xeno sample were compared with their relative expression in $\mathrm{N}$-xeno sample, and those with at least 1.5 -fold higher relative expression in R-xeno TISEs are shown as fold enrichment. Expression in each sample normalized to the internal median value is shown, along with the relative expression for that microRNA in R-xeno (TISE R-xeno/TISE N-xeno).

nal at all tested postrejection time points on NanoSight fluorescence (Figure 7G), and this was also validated on Western blot (Figure 7H).

Taken together, these findings demonstrate that in clinical islet transplantation, donor islet exosome quantitation and characterization can be successfully performed from recipient plasma using donor HLA-specific antibodies. Similarly to the xenoislet model, donor islet exosomes coexpress islet surface markers and their cargoes are specific to the cellular constituents of the transplanted islets.

Transplant tissue-specific exosomes can be characterized in clinical renal transplantation. To test whether our proposed concept can be used to study other transplant tissue types and whether transplant tissue exosomes can be profiled from other bodily fluids, we assessed for donor kidney-specific exosomes in the plasma and urine samples of patients undergoing living-donor renal transplantation $(n=5)$. Representative results from a single donor-recipient pair are shown (donor: HLA-A2, HLA-B27; recipient: HLA-A29, HLA-A31, HLA-B44). On NanoSight fluorescence, post-transplant recipient plasma samples showed donor kidney-specific HLA-A2and HLA-B27-positive exosomes (Supplemental Figure 4). Western blot analysis of post-transplant plasma HLA-A2-bound exosomes confirmed expression of the renal epithelial protein aquaporin 2 (Supplemental Figure 4). These findings were also validated in recipient urine exosomes, where post-transplant urine sample showed donor HLA-A2 exosomes on NanoSight fluorescence (Supplemental Figure 4), and anti-HLA-A2 antibody bead-bound urinary exosomes from post-transplant days 4 and 30 showed presence of the renal glomerular protein podocalyxin-1 (Supplemental Figure 4).

In the field of kidney transplantation, studies have shown elevated T cell RNA signatures in recipient urinary cell pellet as a marker for acute rejection (32-36). Therefore, we assessed the feasibility of characterizing (CD3-positive) $\mathrm{T}$ cell-specific exosomes from recipient urine, which may also serve as a marker of rejection. First, we noted that CD3-positive exosomes could be detected among recipient urine HLA-A2-unbound exosomes in the post-transplant samples but not the pretransplant sample (Supplemental Figure 4). Using anti-CD3 antibody-conjugated beads we purified a $\mathrm{T}$ cell exosome subset, and validated that it was highly positive for surface coexpression of Th cell (CD4) and cytotoxic T cell (CD8) markers (Supplemental Figure 4). Further, HLA-A2-unbound, CD3-unbound urine exosomes were positive for the B cell surface marker CD19 (Supplemental Figure 4). Taken together these findings support the concept that donor tissue-specific and $\mathrm{T}$ and $\mathrm{B}$ cell-specific exosome characterization can be performed with other transplantation tissue types, and from other bodily fluids in continuity with the transplanted organ.

\section{Discussion}

Exosomes have not been as extensively studied in the field of transplantation. We investigated the diagnostic potential of transplant tissue-specific exosome characterization as a noninvasive biomarker to monitor rejection. To our knowledge this is one of the first demonstrations that (a) transplant tissue releases donor HLAspecific exosomes into recipient circulation, (b) transplant exosomes can be quantified and profiled noninvasively and tracked over long-term follow-up, (c) immunologic rejection leads to distinct changes in the exosome signal quantity in a time-specific manner, and (d) RNA and proteomic signatures of transplant exosomes are also tissue specific, and change as a result of rejection. For these reasons, we believe that transplant exosome profiles can serve as a liquid biopsy of the conditional status of the transplant tissue, and this investigation is the first step in validation of this concept.

Our study demonstrates that transplant exosome profiling has potential to markedly improve diagnostic accuracy compared 


\section{A}

1. Ugl-Y3 OS=Homo sapiens $\mathrm{GN}=\mathrm{FN} 1 \mathrm{PE}=2 \mathrm{SV}=1$

2. Vimentin $\mathrm{OS}=\mathrm{Homo}$ sapiens $\mathrm{GN}=\mathrm{VIM} P E=3 \mathrm{SV}=1$

3. Myosin regulatory light chain 12A OS=Homo sapiens $\mathrm{GN}=\mathrm{MYL} 12 \mathrm{APE}=4 \mathrm{SV}=1$

4. Myosin light chain $3 \mathrm{OS}=$ Homo sapiens $\mathrm{GN}=\mathrm{MYL} 3 \mathrm{PE}=1$ $\mathrm{SV}=3$

5. Alpha-crystallin $B$ chain $O S=$ Homo sapiens $G N=C R Y A B$ $P E=1 S V=2$

6. Filamin-A $\mathrm{OS}=\mathrm{Homo}$ sapiens $\mathrm{GN}=\mathrm{FLNA} \mathrm{PE}=2 \mathrm{SV}=1$

7. Ubiquitin $\mathrm{OS}=\mathrm{Homo}$ sapiens $\mathrm{GN}=\mathrm{UBB} \mathrm{PE}=2 \mathrm{SV}=1$

8. Aldehyde dehydrogenase family 16 member $A 1 \mathrm{OS}=\mathrm{Homo}$ sapiens $\mathrm{GN}=\mathrm{ALDH} 16 \mathrm{~A} 1$

9. Isoform 3 of Histone-lysine $\mathrm{N}$-methyltransferase $2 \mathrm{C}$ $\mathrm{OS}=$ Homo sapiens $\mathrm{GN}=\mathrm{KMT} 2 \mathrm{C}$

10. Kininogen $-1 \mathrm{OS}=$ Homo sapiens $\mathrm{GN}=\mathrm{KNG} 1 \mathrm{PE}=1 \mathrm{SV}=2$

11. Antithrombin-III OS=Homo sapiens $\mathrm{GN}=\mathrm{SERPINC} 1 \mathrm{PE}=1$ $\mathrm{SV}=1$

12.Isoform 2 of Liver carboxylesterase $1 \mathrm{OS}=$ Homo sapiens $\mathrm{GN}=\mathrm{CES} 1$

B

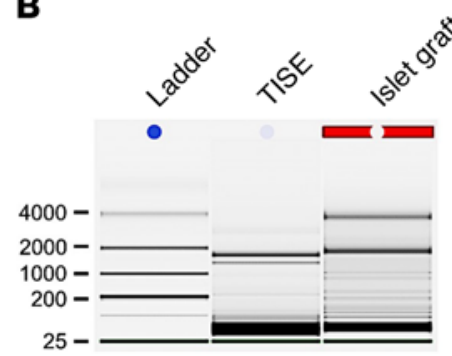

N-Xeno 1

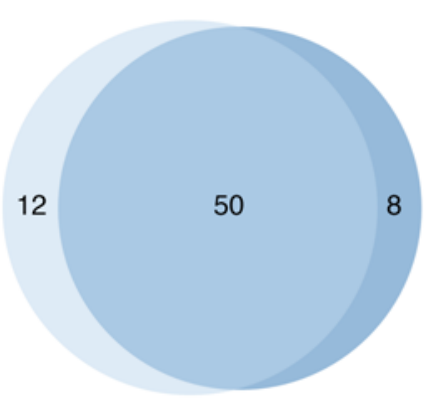

1. Isoform 5 of Fibronectin OS=Homo sapiens $\mathrm{GN}=\mathrm{FN} 1$

2. Pregnancy zone protein $\mathrm{OS}=$ Homo sapiens $\mathrm{GN}=\mathrm{PZP} P E=1 \mathrm{SV}=4$

3. Brain-specific angiogenesis inhibitor 1 associated protein 2 OS=Homo sapiens $\mathrm{GN}=\mathrm{BAIAP} 2 \mathrm{PE}=1 \mathrm{SV}=1$

4. Proteasome subunit alpha type-2 OS=Homo sapiens $\mathrm{GN}=\mathrm{PSMA} 2 \mathrm{PE}=1 \mathrm{SV}=2$

5. Prothrombin $\mathrm{OS}=\mathrm{Homo}$ sapiens $\mathrm{GN}=\mathrm{F} 2$ $P E=1 S V=2$

6. Malate dehydrogenase, mitochondrial $\mathrm{OS}=$ Homo sapiens $\mathrm{GN}=\mathrm{MDH} 2 \mathrm{PE}=2 \mathrm{SV}=1$

7. Collagen alpha-1(IV) chain $\mathrm{OS}=\mathrm{Homo}$ sapiens $\mathrm{GN}=\mathrm{COL} 4 \mathrm{~A} 1 \mathrm{PE}=1 \mathrm{SV}=3$

8. Isoform 2 of Dermcidin $\mathrm{OS}=$ Homo sapiens $\mathrm{GN}=\mathrm{DCD}$
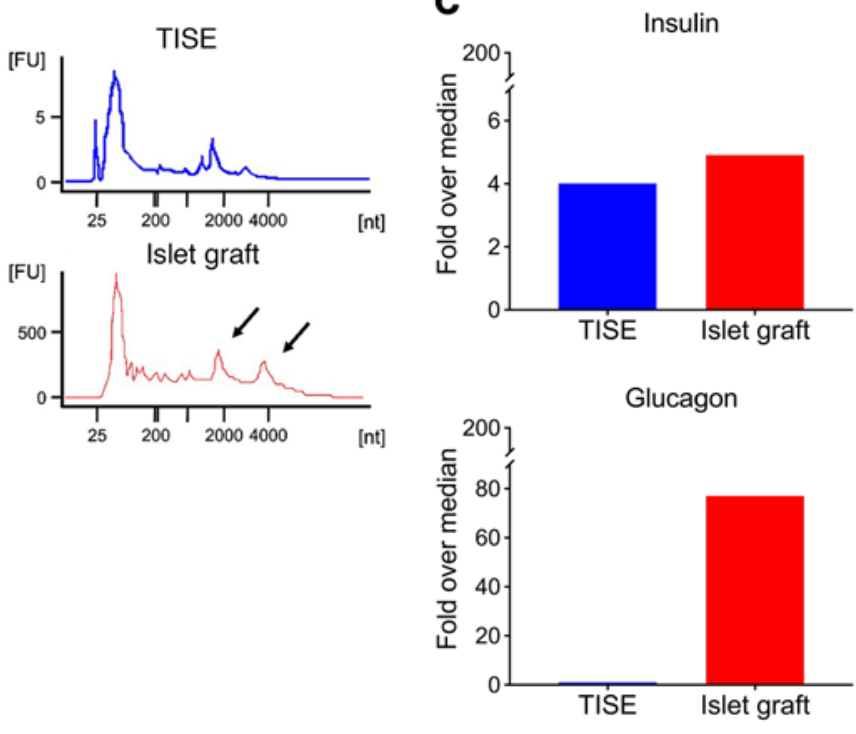

C
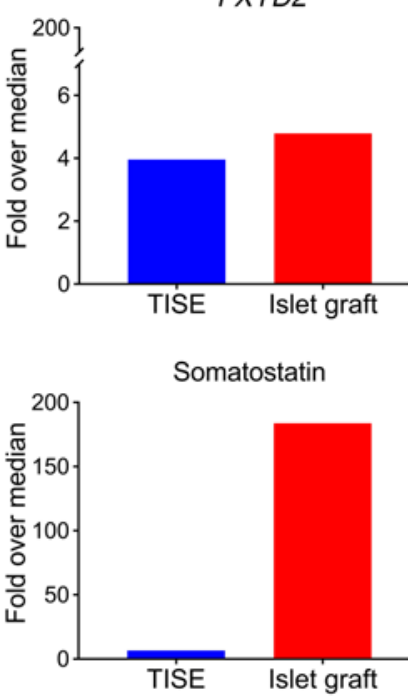

Figure 4. Proteomic and RNA profiling of TISEs. (A) Hybrid mass spectrometry results of transplant islet exosome protein cargoes from 2 independent experiments are shown (labeled $\mathrm{N}$-xeno 1 and 2 ). The proteins seen in $\mathrm{N}$-xeno 1 (12) but not seen in $\mathrm{N}$-xeno2, and vice versa (8 proteins in $\mathrm{N}$-xeno 2 ), are listed. In comparing differences between $\mathrm{N}$-xeno and R-xeno proteomic profiles, only the 50 common proteins in the $2 \mathrm{~N}$-xeno samples were used, with confirmation that they are human derived. (B) RNA gel for TISEs and islet graft is shown, along with the RNA size distribution. TISEs showed higher amounts of small RNAs compared with the islet graft, and the $28 \mathrm{~S}$ and $18 \mathrm{~S}$ ribosomal RNA peaks in islet graft (arrow) were not noted in TISEs. FU, fluorescence units. (C) Long RNA profiles of TISEs and islet graft were obtained using Affymetrix Human Gene 2.0 ST Array. TISEs contained low levels of glucagon and somatostatin, but similar levels of insulin and FXYD2, compared with the islet graft. Expression is shown as fold enrichment over the median value for the particular microarray.

with whole plasma analysis of exosomes. In support of this idea, in the xenoislet model total plasma exosome numbers and exosome particle size were unchanged with rejection, but the transplant exosome signal significantly decreased during early rejection (Figure 2). The high diagnostic accuracy of this platform is further validated by the ability to noninvasively enrich for exosomes containing the cellular constituents of the transplanted human islets in both animal and clinical islet transplant settings. In terms of its time sensitivity, in the xenoislet model we noted that transplant islet exosome profiling is a far more accurate noninvasive platform than glucose monitoring parameters. Future studies investigating changes in the transplant exosome proteomic and RNA cargoes during the early time period of acute rejection will help to further understand its diagnostic accuracy. Also, different changes in the transplant exosome cargoes may be associated with different types of injury, such as acute cellular versus antibody-mediated rejection, or transplant organ infection. In the clinical setting, we noted a time-sensitive change in transplant islet exosome signal in the subject who developed autoimmune recurrence of type 1 diabetes with ensuing hyperglycemia 6 months later. The drop in the exosome signal in this patient temporally correlated with the rise in anti-islet autoantibody titer, when the patient was still normoglycemic and insulin independent (Figure 6).

In addition to quantitative analysis of transplant exosome signal, our proposed concept enables detailed characterization of the intra-exosomal proteomic and RNA cargoes. We believe that this will improve the diagnostic accuracy of the proposed platform. In the xenoislet model, we found specific changes in the proteom- 
A
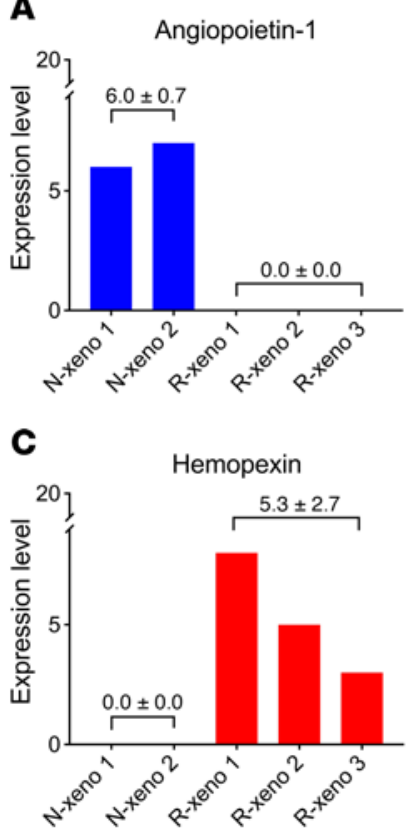

B

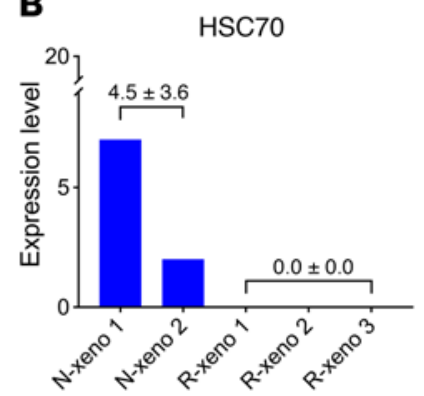

D

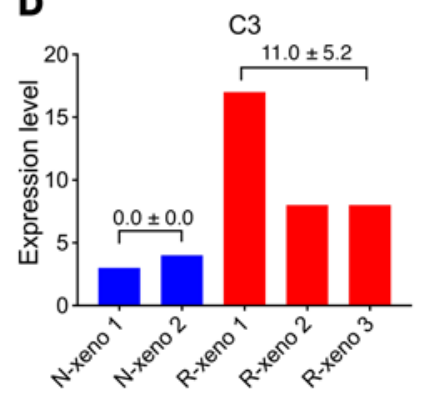

Figure 5. Differences in proteins expressed in TISEs from $\mathbf{N}$-xeno versus R-xeno samples. Proteomic data from mass spectrometry from $2 \mathrm{~N}$-xeno and $3 \mathrm{R}$-xeno TISE samples were analyzed for consistent differences between the 2 groups. This revealed 4 proteins, angiopoietin-1 (A), HSC70 (B), hemopexin (C), and complement C3 (D), to be differentially expressed. Quantitative normalized values are shown.

ic and RNA profiles with the onset of clinical rejection (Table 3). Of the 4 human proteins noted to be different in conditions of acceptance versus rejection, angiopoietin-1 and heat shock protein $71 \mathrm{kDa}$, which were seen only with acceptance, have both been reported to play important protective roles in islet physiology. Angiopoietin-1 production in islets improves revascularization after transplantation and protects from cytokine-induced apoptosis (37). With islet rejection, we noted complete absence of angiopoietin-1 and heat shock protein $71 \mathrm{kDa}$, but found elevated levels of complement C3 and hemopexin (Figure 5). C3 expression on donor tissue has been shown to be necessary for $\mathrm{T}$ cell-mediated transplant organ rejection (38), and elevated hemopexin levels have been seen in donor graft tissue under conditions of rejection $(39,40)$. Further, microarray analysis showed differences in RNA cargoes with rejection. There was upregulation of several microRNAs with rejection compared with acceptance; but interestingly, the majority of microRNAs were common under both conditions. These data suggest that transplant islet exosome purification even at low levels of donor HLA signal can be reliably performed, or else one would expect much more variation between the 2 samples. These findings strongly support future investigations of this platform in animal and clinical transplantation models.

We recognize that other noninvasive biomarker platforms have been reported in the context of transplant rejection versus acceptance (41-44). Recipient plasma cellular gene expression profiling has been studied to monitor for rejection in heart transplant patients (41), and currently, the AlloMap (Care Dx, Inc) is the only commercially available noninvasive platform for heart transplant patients. This assay is validated for ruling out moder- ate to severe rejection between 6 months and 5 years after heart transplantation, and the specificity of this assay is a concern, as the majority of the gene expression profiles are related to the recipient immune cell response. Similarly, exosome shuttle RNA profiles from bronchoalveolar lavage samples in lung transplant patients have been reported (43). Recently, cell-free DNA analysis from recipient serum has gained increasing attention (44-48), with greater focus on quantitative changes in donor-derived cellfree DNA (ddcfDNA) levels as a biomarker for monitoring moderate or greater rejection. This technique is appealing because it has potential to be universally applied across all transplant organ types, but ddcfDNA represents a very low fraction of the total serum cell-free DNA (0.06\%-3.5\%) (49-51), it can be associated with high background, it may not distinguish among transplant organ rejection types or rejection versus infection, and its accuracy may vary based on patient age.

Our proposed idea conceptually includes all the advantages of the above-mentioned biomarker platforms and also may address their associated concerns. Urine exosome analysis in kidney transplant patients demonstrated that transplant exosome analysis can be performed from other bodily fluids. Similar to ddcfDNA analysis, our proposed platform has potential to be universally applied for all transplanted tissues. Also, exosomes have been shown to express DNA fragments inside them $(52,53)$. It might be important to know whether transplant exosomes carry donor-derived exosomal DNA as part of their cargo, especially under conditions of transplant rejection versus acceptance. If so, its quantitation may add another level of improved diagnostic accuracy. Our laboratory is currently studying this idea in the xenoislet transplant model over a predictable time course of rejection of transplanted human islets.

In addition to its diagnostic potential, the ability to enrich tissue-specific exosome subsets noninvasively also opens a window into understanding their roles in the crosstalk between the recipient immune system and the donor tissue. Studies investigating changes in transplant exosome and $\mathrm{T}$ cell exosome profiles under conditions of tolerance versus acceptance versus rejection in animal models may provide mechanistic insights into transplantation tolerance. Such an understanding may also enable careful titration of immunosuppressive regimen, which would help decrease the risk of infection and malignancy in transplant patients. Therefore, we believe that the described transplant exosome and immune cell exosome platforms may have diagnostic as well as therapeutic implications.

Lastly, another important aspect of our study might be the validation of the accuracy of the donor HLA exosomes for cellular constituents of the transplanted tissue. Given the increasing literature supporting physiologic roles for exosomes in conditions of homeostasis versus disease, our results demonstrating in vivo expression of insulin in islet exosomes suggest a novel mechanism of insulin release by $\beta$ cells through exosomes, implicating their role in glucose regulation and energy metabolism. This idea is further supported by the finding that distinct microRNAs were upregulated in islet exosomes in the xenoislet model. Although most of these microRNAs are not well studied, miR-122, reported to be liver specific (25-30), was highly enriched in transplant exosomes (1,060-fold). miR-122 has pro-insulinogenic effects in hepatocytes by upregulating hepatocyte lipid and cholesterol synthesis, and inhibiting gluconeogenesis. The $\beta$ cell-specific microRNA miR-375 $(23,24)$, which inhibits insulin release, was 
A

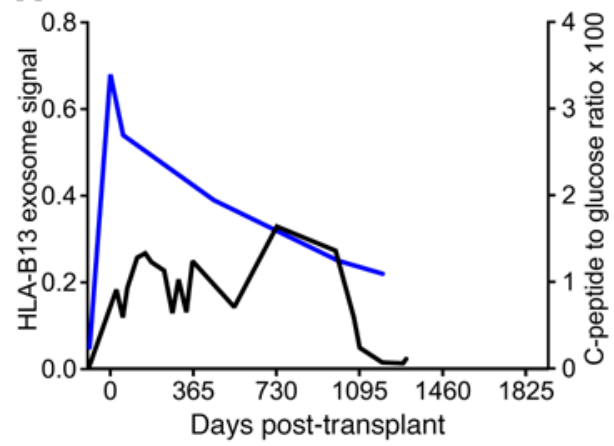

C

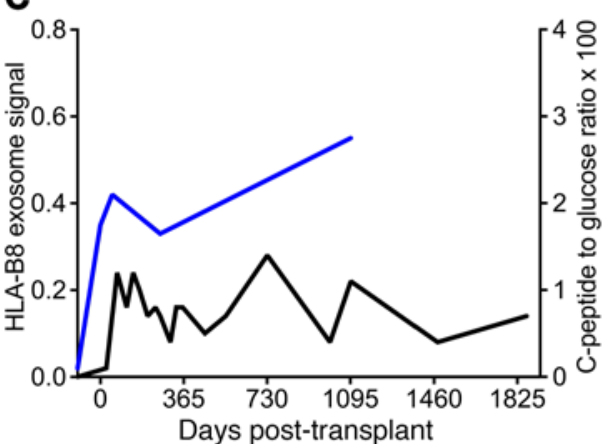

$\mathbf{E}$

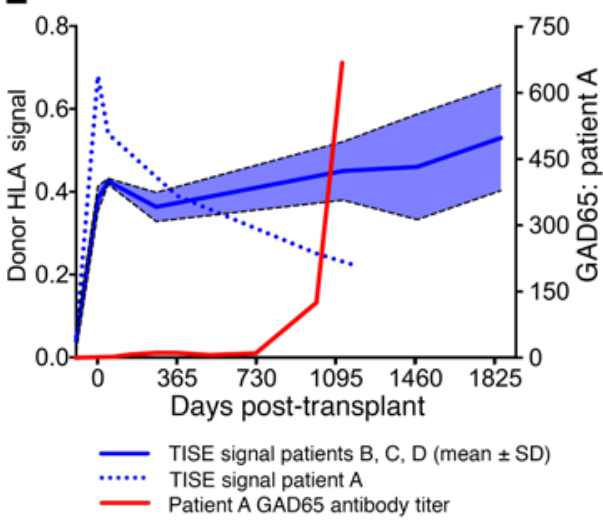

B

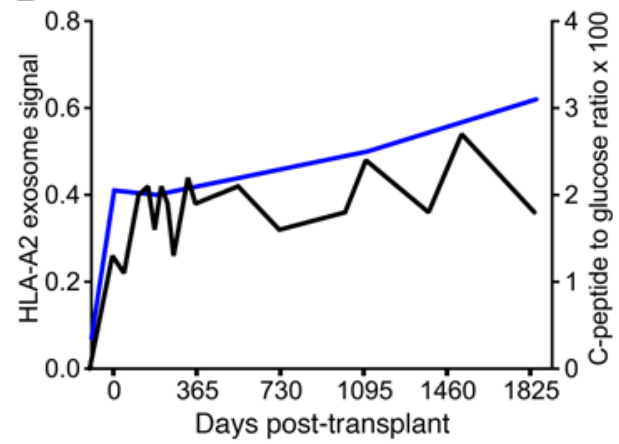

D

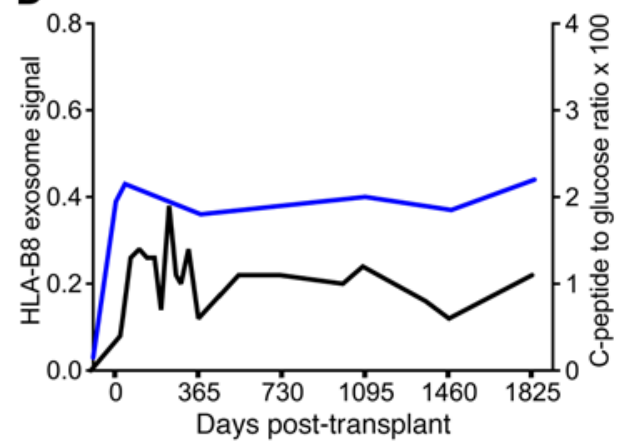

Figure 6. Quantitation of transplant islet exosome signal in human allogeneic islet transplantation over long-term follow-up. (A-D) Plasma samples from islet transplant recipients, patients A-D, were analyzed on NanoSight using anti-donor HLA class I-specific antibody quantum dot, and the transplant islet exosome signal (primary $y$ axis, blue line) was quantified over long-term follow-up (up to 1,848 days after transplant). In all 4 patients, the pretransplant sample showed donor HLA exosome signal equivalent to the IgG isotype, but all the post-transplant samples reliably showed long-term tracking of the donorspecific HLA exosome signal $(P=0.0001)$. Recipient plasma [C-peptide $(\mathrm{ng} / \mathrm{ml})$ to glucose $(\mathrm{mg} / \mathrm{dl})$ ratio] $\times 100$ values over the follow-up period are also shown (secondary $y$ axis, black line). (E) Mean transplant islet exosome signal in patients B-D is shown along with the signal in patient $A$ separately, as the latter subsequently developed diabetes. Unlike patients B-D, who maintained TISE signal greater than 0.35 at all post-transplant time points, patient A showed progressive loss in TISE signal to below 0.35 by the day 1,001 time point, when the plasma C-peptide-to-glucose ratio was still normal. However, at the day 1,001 time point, patient A developed signs of recurrent autoimmunity as evidenced by rapid increase in the $\beta$ cell autoimmune antibody GAD65 (secondary $y$ axis). This patient subsequently developed hyperglycemia requiring exogenous insulin therapy by the day 1,198 time point (Supplemental Table 2).

markedly downregulated in islet exosomes compared with the islet graft (3,285-fold enriched in islet graft). miR-3613-5p, the most upregulated islet exosome microRNA (1,843-fold), is predicted to bind targets such as MBNL2, an RNA-binding protein mediating pre-mRNA alternative splicing and expression, including insulin receptor isoforms $(35,54)$ (www.targetscan.org, www.mirdb.org). Given these data, it is tempting to speculate that highly enriched RNA and protein cargoes in $\beta$ cell exosomes have physiologic functions in mediating differential insulinogenic effects on target tissues and in regulating local paracrine effects in coordinating islet physiology and function. We believe that future in vivo studies exploring $\beta$ cell exosome biology using the xenoislet model may also provide deeper insights into the physiology of energy metabolism, nutridynamics, and disease states such as diabetes and metabolic syndrome. Further, islet $\beta$ cellspecific exosome characterization from patient plasma may facilitate development of noninvasive biomarker platforms for detection, 
A

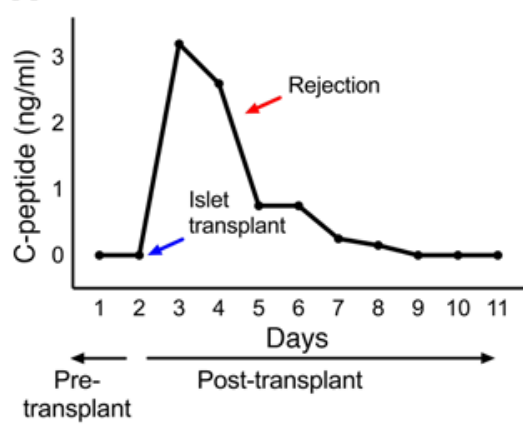

B

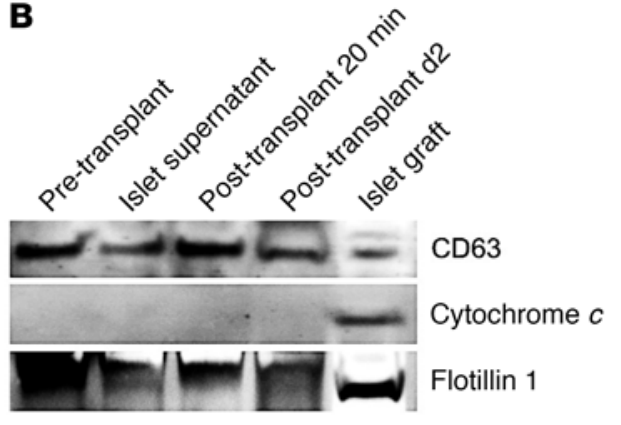

D

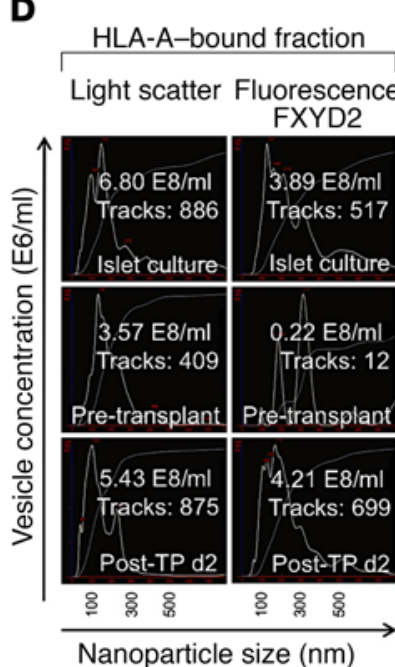

G

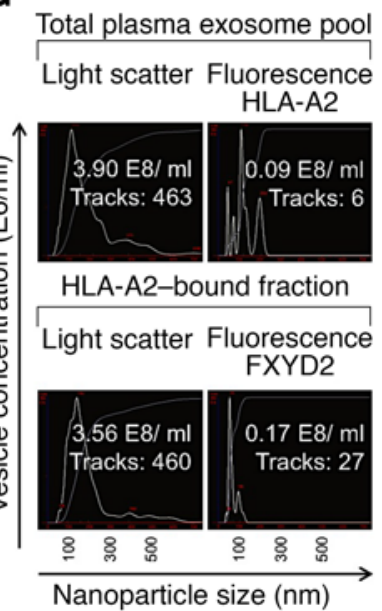

E

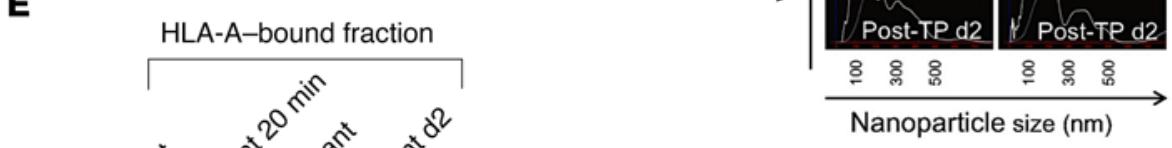

C

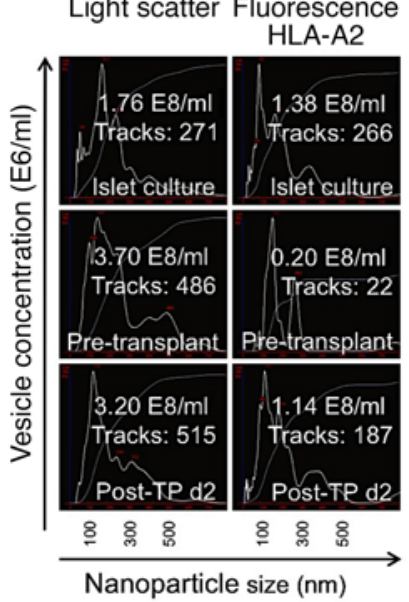

$\mathbf{F}$

HLA-A-bound fraction

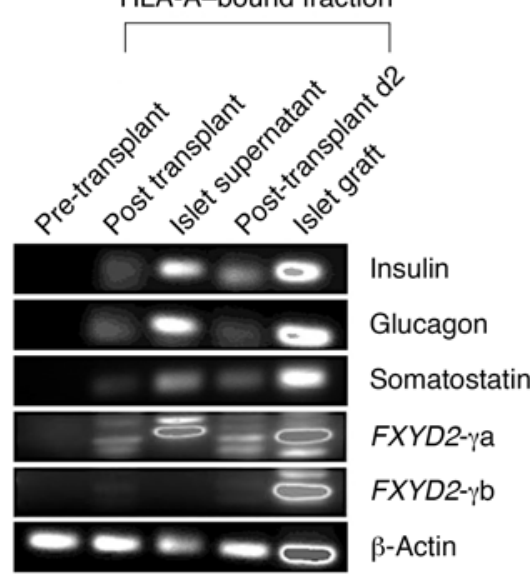

H

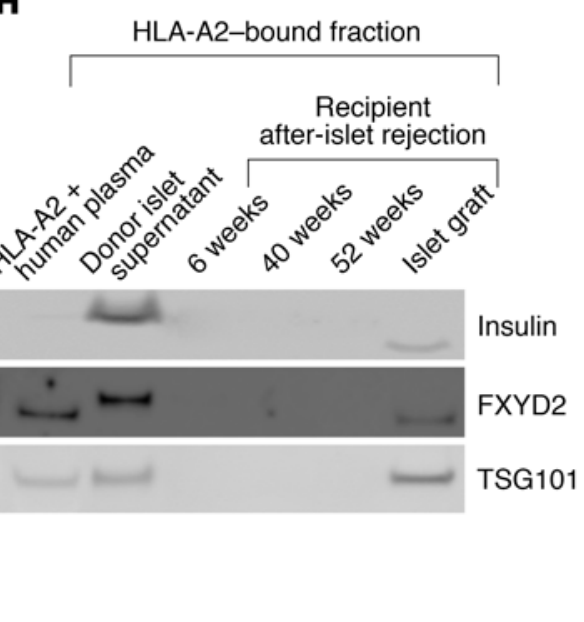

Figure 7. TISE cargoes can be characterized from recipient plasma in human allogeneic islet transplantation. TISEs were purified from plasma using anti-donor HLA class I-specific antibodies in patients A-E $(n=5)$. Representative results in patient $E$ are shown. (A) C-peptide production in the peritransplant period is shown. The patient had acute rejection of the donor islets, with rise in alloantibody titer and drop in C-peptide by day 5. (B) As confirmation that the isolated microvesicles contained exosomes, samples tested on Western blot showed exosome markers CD63 and flotillin 1, but absence of cellular/ apoptotic body marker cytochrome $c$. (C) NanoSight fluorescence for donor-specific HLA-A2 signal demonstrated TISEs on post-transplant day 2, but not in pretransplant sample. Donor islet culture exosomes are used as positive control ( $P=0.008 ; 1$ of 5 shown). (D) FXYD2 coexpression in HLA-bound exosomes showed high signal in donor islet culture and recipient post-transplant day 2 samples, but not the pretransplant sample. (E) Western blot of HLA-A2-bound exosomes showed islet endocrine hormone protein and FXYD2 expression in post-transplant day 2 sample but not pretransplant sample $(P=0.008)$. Islet graft obtained from xenoislet experiments served as positive tissue control. TSG101 is an exosome marker. (F) RT-PCR showed islet endocrine hormones and

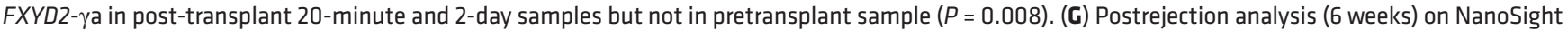
for HLA-A2 in recipient plasma (top) and for FXYD2 in HLA-A2-bound fraction (bottom) were both negative. (H) Western blot analysis of HLA-A2-bound fractions from recipient plasma after rejection showed absence of insulin and FXYD2. Normoglycemic HLA-A2-positive blood donor (HLA-A2-positive human plasma) is shown to validate that the bead-bound exosomes were not nonspecifically binding islet exosomes $(P=0.008 ; 1$ of 5 shown). 
monitoring, and prognostication of functional $\beta$ cell mass in diseases such as metabolic syndrome and types 1 and 2 diabetes. Therefore, a transplant tissue-specific exosome platform might have applications in understanding the role of exosomes in other fields using xenograft/ allograft models, such as cancer biology and stem cell biology.

In conclusion, we demonstrate that transplant tissue-specific exosome characterization can be successfully performed from recipient plasma/bodily fluids. Transplant exosome quantity and intra-exosomal cargo reflect immune condition-specific changes to the transplant tissue. We have shown the translational potential of this platform in the clinical settings of human allogeneic islet and renal transplantation. These findings warrant further detailed investigations into the diagnostic potential of the transplant tissue exosome platform.

\section{Methods}

Xenoislet transplantation-related procedures and post-transplant monitoring methods are described in Supplemental Methods.

Human plasma and urine sample analysis. University of Pennsylvania Institutional Review Board approval was obtained for collection and analysis of human plasma and urine samples. Informed patient consent was obtained in each case. For each time point, 0.25-1 ml of plasma was obtained through venipuncture and stored at $-80^{\circ} \mathrm{C}$. Urine samples $(40 \mathrm{ml})$ were collected in sterile cups, treated with $1^{\prime}$ protease inhibitor cocktail (Sigma-Aldrich), and frozen at $-80^{\circ} \mathrm{C}$ for later analysis. Human islet transplant patient samples were provided through the NIH Beta Cell Consortium (Bethesda, Maryland, USA).

Human pancreatic islet isolation and islet transplantation. Human pancreas was processed for islet isolation, and high-purity (>80\%) islets were used for xenoislet transplantation. Islet isolation was performed by the cGMP Islet Isolation Laboratory at the University of Pennsylvania in accordance with approved Institutional Review Board protocols. Islets were cultured in CMRL media supplemented with albumin, without any exogenous exosome contamination. Islet culture supernatant (20 $\mathrm{ml}$ ) was obtained 24-72 hours after isolation for exosome analysis. Clinical islet transplantation was performed at the Hospital of the University of Pennsylvania under Clinical Islet Transplantation (CIT) Consortium protocols CITO7 (Islet alone; ClinicalTrials.gov NCT00434811) and CIT06 (Islet-after-kidney; ClinicalTrials.gov NCT00468117).

Antibodies. All antibodies were commercially available. A table of antibodies used is given in Supplemental Methods.

Exosome isolation. Exosomes were isolated from human islet culture supernatants by size exclusion limit gel chromatography along with ultracentrifugation. Briefly, $10 \mathrm{ml}$ culture supernatant was centrifuged at $500 \mathrm{~g}$ for 10 minutes, and supernatant was then passed through a Sepharose 2B column (Sigma-Aldrich) and the eluent was collected in 1-ml fractions. The exosome fraction was pooled after monitoring of absorbance at $280 \mathrm{~nm}$. The pooled fraction was filtered through a $100-\mathrm{kDa}$-cutoff membrane and then ultracentrifuged at $120,000 \mathrm{~g}$ for 4 hours at $4^{\circ} \mathrm{C}$. The pelleted exosome fraction was resuspended in PBS for downstream analysis. Mouse and human plasma exosome isolation was performed using $200 \mu \mathrm{l}$ to $1 \mathrm{ml}$ plasma obtained after centrifugation of the blood sample at $500 \mathrm{~g}$ for 10 minutes. Plasma sample was directly added to the column for exosome isolation as described above.

Urinary exosome isolation was performed as described elsewhere with slight modification $(55,56)$. Briefly, sample was centrifuged at 17,000 $g$ for 15 minutes. The supernatant was ultracentrifuged at 200,000 $g$ for
120 minutes at $4^{\circ} \mathrm{C}$. The pellet was resuspended in PBS and loaded onto a Sepharose 2B size exclusion column, and the eluted fractions representing exosomes were pooled. The pooled fractions were concentrated on an Amicon filter (Merck Millipore Ltd.) with a 100-kDa-cutoff membrane.

NanoSight exosome analysis. Exosomes were analyzed on the NanoSight NS300 (405 nm laser diode) on the light scatter mode for quantification and scatter distribution according to the manufacturer's protocols (Malvern Instruments Inc.). Before each experimental run, the machine was calibrated for nanoparticle size and quantity using standardized nanoparticle dilutions provided by the manufacturer. Surface marker detection on exosomes was performed using the fluorescence mode on the NanoSight NS300. Secondary antibodies conjugated to quantum dots with emission at $605 \mathrm{~nm}$ were used for fluorescence detection against specific surface proteins as described previously $(57,58)$. Each experimental run was performed in duplicate, and the appropriate IgG isotype control fluorescence was performed to assess background. In each panel, the nanoparticle size distribution curve represented by particle size (nanometers) ( $x$ axis) and the relative concentration of a nanoparticle $\left(10^{6}\right.$ per milliliter) $(y$ axis) of particular size are shown, with the cumulative percentage of nanoparticles at a certain size cutoff (secondary $y$ axis). The panels shown in the figures are graphical readouts produced by the NanoSight program, NTA 2.3, where the software generated panels with a dynamic $y$ axis for exosome concentration. Therefore, the height of the $y$ axis is automatically normalized by the program to produce graphs of the same height. For this reason, the area under the nanoparticle curve looks very similar for scatter and fluorescent modes. For this reason, we chose to include in each panel the overall particle concentration as listed on the PDF file generated by the NanoSight software.

The number of tracks in scatter mode is an important consideration before interpretation of the data because the NanoSight machine actually tracks each individual particle as it flows across the video screen that tracks the particle. For a reliable scatter and especially fluorescence analysis, it is important that the machine reliably tracks at least 150-200 particles per sample in the scatter mode. Further, the size distribution curve of the tracked particles is important because it is critical that the nanoparticles displayed in the panel are not just protein aggregates but show a size distribution pattern consistent with microvesicles, especially exosomes (30-200 $\mathrm{nm}$ range primarily). Similar evaluation of the fluorescence mode panels is important to confirm that the nanoparticles displayed are not quantum dot aggregates. In this context, it is helpful to have a dynamic $y$ axis that shows particle size distribution, because if the $y$ axis is kept as an absolute scale, then for fluorescence of exosomes in low relative quantities it would make size distribution analysis difficult and may lead to false positives. For these reasons, also written in text inside each panel are the total particles detected $\left(10^{8}\right.$ per milliliter) and the number of particles reliably tracked by NanoSight (Tracks) for each sample. For light scatter mode this represents the total number of nanoparticles, and for fluorescence mode this represents the number of nanoparticles with detected fluorescence signal for the particular antibody used. For every experiment, we attempted to analyze only samples where more than 200 nanoparticles were reliably tracked on the light scatter mode by the NanoSight detector.

HLA-specific exosome signal was quantified as follows:

HLA fluorescence - pretransplant HLA fluorescence - IgG isotype fluorescence HLA light scatter pretransplant light scatter IgG isotype light scatter

(Equation 1) 
Affinity antibody-coupled bead purification of tissue-specific exosomes. Anti-HLA-A antibody was covalently conjugated to $\mathrm{N}$ hydroxysuccinimide magnetic beads (Pierce) per manufacturer's protocol. Fifty to $100 \mu \mathrm{g}$ protein equivalent of exosomes was incubated with antibody beads overnight at $4^{\circ} \mathrm{C}$. The bead-bound and unbound exosome fractions were separated per manufacturer's protocol. Exosomes bound to beads were eluted using Tris glycine and used for downstream analysis.

RNA and protein isolation. RNA was extracted from cells and exosomes using Trizol, followed by RNeasy Mini kit, according to manufacturer's protocol (Qiagen). For total protein isolation, exosomes were lysed in $1^{\prime}$ RIPA buffer with $1 \times$ concentration of protease inhibitor cocktail (Sigma-Aldrich).

Western blot. Exosomes and cell lysate total proteins were isolated and separated on polyacrylamide gels, transferred on PVDF membrane (Life Technologies). The blot was blocked and incubated with the desired antibody at concentration per manufacturer's protocol. HRP-coupled secondary antibody (Santa Cruz Biotechnology Inc.) was added and detected through chemiluminescence using ImageQuant LAS 400 Phospho-Imager.

$R T-P C R$. Total RNA (12.5-50 ng) was reverse transcribed with the SuperScript III 1-step RT-PCR system (Life Technologies) for gene expression validation. The primers used in this study were as follows: human insulin (forward) 5'-CCTTGTGAACCAACACCTG-3', (reverse) 5'-GTAGAAGAAGCCTCGTTCCC-3' (80 bp); human glucagon (forward) 5'-CCCAAGATTTTGTGCAGTGGTT-3', (reverse) 5'-CAGCATGTCTCTCAAATTCATCGT-3' (80 bp); human somatostatin (forward) 5'-GATGCCCTGGAACCTGAAGA-3', (reverse) 5'-CCGGGTTTGAGTTAGCAGATCT-3' (82 bp); human FXYD2ra (forward) 5'-ACTGGGTTGTCGATGGACGGT-3', (reverse) 5'-CGGCTCATCTTCATTGATTTG-3' (188 bp); human FXYD2$\gamma \mathrm{b}$ (forward) 5'-GACAGGTGGTACCTG-3', (reverse) 5'-CGGCTCATCTTCATTGATTTG-3' (188 bp); and human $\beta$-actin (forward) 5'-CTGTACGCCAACACAGTGCT-3'， (reverse) 5'-GCTCAGGAGGAGCAATGATC-3' (127 bp).

Transmission electron microscopy. Exosomes suspended in PBS were processed at the Electron Microscopy Resource Laboratory, University of Pennsylvania, using the standard protocols. Briefly, $50 \mu \mathrm{l}$ of exosomes was absorbed onto Formvar (Polysciences, Inc.) carbon-coated nickel grid for 1 hour. Then the grids were sequentially washed with $0.1 \mathrm{M}$ sodium cacodylate, $\mathrm{pH} 7.6$, and fixed in $2 \%$ paraformaldehyde and $2.5 \%$ glutaraldehyde in $0.1 \mathrm{M}$ sodium cacodylate, contrasted with $2 \%$ uranyl acetate in $0.1 \mathrm{M}$ sodium cacodylate, for 15 minutes. After another washing, grids were incubated with $0.13 \%$ methyl cellulose and negatively stained with $0.4 \%$ uranyl acetate for 10 minutes, air-dried, and visualized under the JEM-2200FS transmission electron microscope operated at $100 \mathrm{kV}$.

RNA microarray. Samples were analyzed using the Agilent 2100 Bioanalyzer and Nanodrop spectrophotometry at the Molecular Profiling Facility, University of Pennsylvania. All protocols were performed according to the NuGEN Ovation Pico WTA system version 2 user guide and the Affymetrix GeneChip Expression Analysis Technical Manual (see Supplemental Methods). Microarray profile data files were submitted to NCBI Gene Expression Omnibus (GSE888460).

Proteomic profiling. $\mathrm{N}$-hydroxysuccinimide-HLA-A-conjugated bead-bound exosomes $(1 \mu \mathrm{g})$ were eluted in $50 \mu \mathrm{l}$ volume of Tris-glycine and digested with trypsin, analyzed with nano-Ultraperformance liquid chromatography - tandem mass spectrometry on the Orbitrap Elite hybrid mass spectrometer (Thermo Fisher Scientific) at the Penn Proteomic Core, University of Pennsylvania. The data were analyzed with $\mathrm{PD} /$ Scaffold software package to search human protein databases, with the following cutoffs: peptide confidence value less than $95 \%$ and protein confidence value less than $99 \%$. Total spectrum count values as displayed by the software program were used for quantitation.

Statistics. Kolmogorov-Smirnov test was used to characterize distribution. Statistical significance for parametric data was assessed by unpaired Student's $t$ test (2-tailed) for continuous variables with 2 groups and ANOVA with post hoc Tukey test for pairwise comparison of subgroups for continuous variable with more than 2 groups (equal variance assumed). The Pearson's $\chi^{2}$ test was used for categorical variables. All reported $P$ values are 2 -sided and are considered statistically significant if below 0.05. For receiver operating characteristic (ROC) curve, the true-positive rate (sensitivity) was plotted against the false-positive rate (specificity) to illustrate performance of a binary classifying system (rejection versus nonrejection). A threshold was determined using the Youden index, and sensitivity and specificity were calculated. ROC curves were compared using the method of Delong et al. (59). General statistics were assessed using SPSS version 23 (Armonk), and scatter plots were constructed using Prism version 7.0 (GraphPad). Sample size calculations were done using G Power version 3.1 (University of Düsseldorf, Düsseldorf, Germany). Area-proportional Venn diagrams were calculated and drawn using the BioVenn web application (http://www.cmbi.ru.nl/cdd/biovenn).

Study approval. Animal studies and analysis of human plasma and urine samples were approved by the University of Pennsylvania Institutional Review Board. Subjects provided informed consent prior to participation in the study. Analysis of plasma samples from islet transplant patients was approved by the NIH Beta Cell Consortium (Bethesda, Maryland, USA).

\section{Author contributions}

LK performed all the exosome experiments involving characterization and profiling of exosome cargo. MY and S. Rostami performed the animal experiments (provided by AN). S. Reddy, AH, and VK helped with exosome isolation and characterization. $\mathrm{AH}$ performed statistical analysis. CXY helped with proteomic profiling and analysis of exosomes. CL performed islet isolation and culture studies. BK performed islet histology. JTC assisted with human sample collection, processing, and patient consent, and obtained ethical approval of human studies. MRR and AN performed human islet transplants and assisted with sample collection and patient data analysis and critical review of the manuscript. PV conceived the proposed diagnostic platform and performed experimental design for all the experiments, drafted and edited the manuscript, supervised all the experiments, and in conjunction with LK established and optimized tissue-specific exosome characterization protocols, NanoSight exosome analysis, and exosome RNA and proteomic profiling experiments.

\section{Acknowledgments}

We thank the proteomics and RNA core facilities at the University of Pennsylvania for assistance with the proteomic and 
microarray profiling, and Heather Collins for performing C-peptide ELISA. We thank David Sachs at Columbia University for critical review of the manuscript. This work was supported by Public Health Service research grant NIH/NIAID R21 AI1101001 (to PV), U01 DK070430 and NIDDK/City of Hope Integrated Islet Distribution Program (to AN), UL1 TR000003 (University of Pennsylvania Clinical \& Translational Research Center), P30 DK19525 (University of Pennsylvania Diabetes Research Cen- ter), and the Department of Surgery, University of Pennsylvania Perelman School of Medicine (to PV).

Address correspondence to: Prashanth Vallabhajosyula, 6 Silverstein, Division of Cardiovascular Surgery, Hospital of the University of Pennsylvania, 3400 Spruce Street, Philadelphia, Pennsylvania 19104, USA. Phone: 215.662.2957; E-mail: Prashanth. vallabhajosyula@uphs.upenn.edu.
1. Yusen RD, et al. The Registry of the International Society for Heart and Lung Transplantation: Thirty-second Official Adult Lung and HeartLung Transplantation Report - 2015; Focus Theme: Early Graft Failure. J Heart Lung Transplant. 2015;34(10):1264-1277.

2. Lund LH, et al. The Registry of the International Society for Heart and Lung Transplantation: Thirty-second Official Adult Heart Transplantation Report - 2015; Focus Theme: Early Graft Failure. J Heart Lung Transplant. 2015;34(10):1244-1254.

3. Goldfarb SB, et al. The Registry of the International Society for Heart and Lung Transplantation: Eighteenth Official Pediatric Lung and Heart-Lung Transplantation Report - 2015; Focus Theme: Early Graft Failure. J Heart Lung Transplant. 2015;34(10):1255-1263.

4. Dipchand AI, et al. The Registry of the International Society for Heart and Lung Transplantation: Eighteenth Official Pediatric Heart Transplantation Report - 2015; Focus Theme: Early Graft Failure. J Heart Lung Transplant. 2015;34(10):1233-1243.

5. Josephson MA. Monitoring and managing graft health in the kidney transplant recipient. Clin J Am Soc Nephrol. 2011;6(7):1774-1780.

6. Farid WR, et al. Hepatocyte-derived microRNAs as serum biomarkers of hepatic injury and rejection after liver transplantation. Liver Transpl. 2012;18(3):290-297.

7. Fleissner F, Goerzig Y, Haverich A, Thum T. Microvesicles as novel biomarkers and therapeutic targets in transplantation medicine. Am J Transplant. 2012;12(2):289-297.

8. Johann DJ Jr. Blonder J. Biomarker discovery: tissues versus fluids versus both. Expert Rev Mol Diagn. 2007;7(5):473-475.

9. Julich H, Willms A, Lukacs-Kornek V, Kornek M. Extracellular vesicle profiling and their use as potential disease specific biomarker. Front Immunol. 2014;5:413.

10. Lakkaraju A, Rodriguez-Boulan E. Itinerant exosomes: emerging roles in cell and tissue polarity. Trends Cell Biol. 2008;18(5):199-209.

11. Lo DJ, Kaplan B, Kirk AD. Biomarkers for kidney transplant rejection. Nat Rev Nephrol. 2014;10(4):215-225.

12. Mathivanan S, Ji H, Simpson RJ. Exosomes: extracellular organelles important in intercellular communication. J Proteomics. 2010;73(10):1907-1920.

13. Matsumoto S, et al. Circulating p53-responsive microRNAs are predictive indicators of heart failure after acute myocardial infarction. Circ Res. 2013;113(3):322-326.
14. Roedder S, Vitalone M, Khatri P, Sarwal MM. Biomarkers in solid organ transplantation: establishing personalized transplantation medicine. Genome Med. 2011;3(6):37.

15. Shan J, et al. MicroRNAs: potential biomarker in organ transplantation. Transpl Immunol. 2011;24(4):210-215.

16. Turchinovich A, Samatov TR, Tonevitsky AG, Burwinkel B. Circulating miRNAs: cell-cell communication function? Front Genet. 2013;4:119.

17. Valadi H, Ekström K, Bossios A, Sjöstrand M, Lee JJ, Lötvall JO. Exosome-mediated transfer of mRNAs and microRNAs is a novel mechanism of genetic exchange between cells. Nat Cell Biol. 2007;9(6):654-659.

18. Lötvall J, et al. Minimal experimental requirements for definition of extracellular vesicles and their functions: a position statement from the International Society for Extracellular Vesicles. JExtracell Vesicles. 2014;3:26913.

19. Arystarkhova E, et al. Hyperplasia of pancreatic beta cells and improved glucose tolerance in mice deficient in the FXYD2 subunit of Na,KATPase. J Biol Chem. 2013;288(10):7077-7085.

20. Flamez D, et al. A genomic-based approach identifies FXYD domain containing ion transport regulator 2 (FXYD2)gammaa as a pancreatic beta cell-specific biomarker. Diabetologia. 2010;53(7):1372-1383.

21. Hald J, et al. Pancreatic islet and progenitor cell surface markers with cell sorting potential. Diabetologia. 2012;55(1):154-165.

22. Wang F, et al. FXYD2, a $\gamma$ subunit of $\mathrm{Na}^{+}$, $\mathrm{K}^{+}$-ATPase, maintains persistent mechanical allodynia induced by inflammation. Cell Res. 2015;25(3):318-334.

23. Poy MN, et al. A pancreatic islet-specific microRNA regulates insulin secretion. Nature. 2004;432(7014):226-230.

24. Poy MN, et al. miR-375 maintains normal pancreatic alpha- and beta-cell mass. Proc Natl Acad Sci US A. 2009;106(14):5813-5818.

25. Hsu SH, et al. Essential metabolic, antiinflammatory, and anti-tumorigenic functions of miR-122 in liver. J Clin Invest. 2012;122(8):2871-2883.

26. Jopling C. Liver-specific microRNA-122: Biogenesis and function. RNA Biol. 2012;9(2):137-142.

27. Lewis AP, Jopling CL. Regulation and biological function of the liver-specific miR-122. Biochem Soc Trans. 2010;38(6):1553-1557.

28. Thakral S, Ghoshal K. miR-122 is a unique molecule with great potential in diagnosis, prognosis of liver disease, and therapy both as miRNA mimic and antimir. Curr Gene Ther. 2015;15(2):142-150.
29. Tsai WC, et al. MicroRNA-122 plays a critical role in liver homeostasis and hepatocarcinogenesis. J Clin Invest. 2012;122(8):2884-2897.

30. Wang K, et al. Circulating microRNAs, potential biomarkers for drug-induced liver injury. Proc Natl Acad Sci U S A. 2009;106(11):4402-4407.

31. Rickels MR, Kamoun M, Kearns J, Markmann JF, Naji A. Evidence for allograft rejection in an islet transplant recipient and effect on beta-cell secretory capacity. JClin Endocrinol Metab. 2007;92(7):2410-2414

32. Aquino-Dias EC, et al. Non-invasive diagnosis of acute rejection in kidney transplants with delayed graft function. Kidney Int. 2008;73(7):877-884

33. Kotsch K, et al. Enhanced granulysin mRNA expression in urinary sediment in early and delayed acute renal allograft rejection. Transplantation. 2004;77(12):1866-1875.

34. Li B, et al. Noninvasive diagnosis of renalallograft rejection by measurement of messenger RNA for perforin and granzyme B in urine. $N$ Engl JMed. 2001;344(13):947-954.

35. Suthanthiran M, et al. Urinary-cell mRNA profile and acute cellular rejection in kidney allografts. N Engl J Med. 2013;369(1):20-31.

36. Tatapudi RR, et al. Noninvasive detection of renal allograft inflammation by measurements of mRNA for IP-10 and CXCR3 in urine. Kidney Int. 2004;65(6):2390-2397.

37. Su D, et al. Angiopoietin-1 production in islets improves islet engraftment and protects islets from cytokine-induced apoptosis. Diabetes. 2007;56(9):2274-2283.

38. Pratt JR, Basheer SA, Sacks SH. Local synthesis of complement component $\mathrm{C} 3$ regulates acute renal transplant rejection. Nat Med. 2002;8(6):582-587.

39. Xu M, et al. Expression of hemopexin in acute rejection of rat liver allograft identified by serum proteomic analysis. Shock. 2014;42(1):65-74.

40. Krikken JA, et al. High plasma hemopexin activity is an independent risk factor for late graft failure in renal transplant recipients. Transpl Int. 2010;23(8):805-812.

41. Deng MC, et al. Noninvasive discrimination of rejection in cardiac allograft recipients using gene expression profiling. Am J Transplant. 2006;6(1):150-160.

42. Gielis EM, et al. Cell-free DNA: An upcoming biomarker in transplantation. Am J Transplant. 2015;15(10):2541-2551.

43. Gregson AL, et al. Altered exosomal RNA profiles in bronchoalveolar lavage from lung transplants with acute rejection. Am J Respir Crit Care Med. 2015;192(12):1490-1503.

44. Snyder TM, Khush KK, Valantine HA, Quake SR. Universal noninvasive detection of solid organ 
transplant rejection. Proc Natl Acad Sci U S A. 2011;108(15):6229-6234.

45. García Moreira V, Prieto García B, Baltar Martín JM, Ortega Suárez F, Alvarez FV. Cell-free DNA as a noninvasive acute rejection marker in renal transplantation. Clin Chem. 2009;55(11):1958-1966.

46. Macher HC, et al. Monitoring of transplanted liver health by quantification of organ-specific genomic marker in circulating DNA from receptor. PLoS One. 2014;9(12):e113987.

47. Sigdel TK, et al. A rapid noninvasive assay for the detection of renal transplant injury. Transplantation. 2013;96(1):97-101.

48. Zhang J, et al. Presence of donor- and recipient-derived DNA in cell-free urine samples of renal transplantation recipients: urinary DNA chimerism. Clin Chem. 1999;45(10):1741-1746.

49. Beck J, et al. Digital droplet PCR for rapid quantification of donor DNA in the circulation of transplant recipients as a potential uni- versal biomarker of graft injury. Clin Chem. 2013;59(12):1732-1741.

50. Botezatu I, et al. Genetic analysis of DNA excreted in urine: a new approach for detecting specific genomic DNA sequences from cells dying in an organism. Clin Chem. 2000;46 (8 Pt 1):1078-1084.

51. De Vlaminck I, et al. Circulating cell-free DNA enables noninvasive diagnosis of heart transplant rejection. Sci Transl Med. 2014;6(241):241ra77.

52. Thakur BK, et al. Double-stranded DNA in exosomes: a novel biomarker in cancer detection. Cell Res. 2014;24(6):766-769.

53. Zhang L, et al. Microenvironment-induced PTEN loss by exosomal microRNA primes brain metastasis outgrowth. Nature. 2015;527(7576):100-104.

54. Ho TH, Charlet-BN, Poulos MG, Singh G, Swanson MS, Cooper TA. Muscleblind proteins regulate alternative splicing. $E M B O J$. 2004;23(15):3103-3112.
55. Pisitkun T, Shen RF, Knepper MA. Identification and proteomic profiling of exosomes in human urine. Proc Natl Acad Sci U S A 2004;101(36):13368-13373.

56. Rood IM, et al. Comparison of three methods for isolation of urinary microvesicles to identify biomarkers of nephrotic syndrome. Kidney Int. 2010;78(8):810-816.

57. Dragovic RA, et al. Sizing and phenotyping of cellular vesicles using Nanoparticle Tracking Analysis. Nanomedicine. 2011;7(6):780-788.

58. Gardiner C, Ferreira YJ, Dragovic RA, Redman $\mathrm{CW}$, Sargent IL. Extracellular vesicle sizing and enumeration by nanoparticle tracking analysis. JExtracell Vesicles. 2013;2:10.3402/jev.v2i0.19671.

59. DeLong ER, DeLong DM, Clarke-Pearson DL. Comparing the areas under two or more correlated receiver operating characteristic curves: a nonparametric approach. Biometrics. 1988;44(3):837-845. 